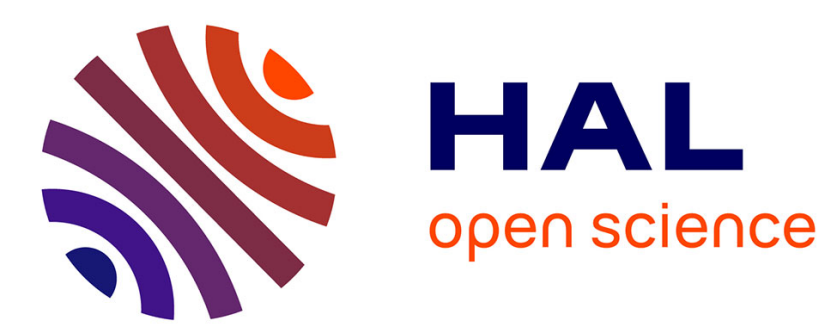

\title{
Influence of the illumination direction on the optical extinction by a scatterer close to a dielectric interface
}

\author{
Michel Pellarin, Jean Lerme
}

\section{To cite this version:}

Michel Pellarin, Jean Lerme. Influence of the illumination direction on the optical extinction by a scatterer close to a dielectric interface. Physical Review A, 2020, 102 (3), pp.033521. 10.1103/PhysRevA.102.033521 . hal-02970785

\section{HAL Id: hal-02970785 \\ https://hal.science/hal-02970785}

Submitted on 5 Nov 2020

HAL is a multi-disciplinary open access archive for the deposit and dissemination of scientific research documents, whether they are published or not. The documents may come from teaching and research institutions in France or abroad, or from public or private research centers.
L'archive ouverte pluridisciplinaire HAL, est destinée au dépôt et à la diffusion de documents scientifiques de niveau recherche, publiés ou non, émanant des établissements d'enseignement et de recherche français ou étrangers, des laboratoires publics ou privés. 


\title{
Influence of the illumination direction on the optical extinction by a scatterer close to a dielectric interface
}

\author{
Michel Pellarin (1)* and Jean Lermé (1) \\ Université de Lyon, Université Claude Bernard Lyon 1, CNRS, Institut Lumière Matière, F-69622 Villeurbanne, France
}

(Received 22 June 2020; accepted 27 August 2020; published 22 September 2020)

\begin{abstract}
The intrinsic optical response of a scattering object is known to be modified when it is placed on a substrate. Moreover, the total extinction through ohmic losses and Rayleigh scattering is basically dependent on the reversal of the direction of the excitation wave. Considering, as stated by the generalized optical theorem, that the total extinction of the incident wave is shared among the extinction of the waves transmitted and reflected by the non-absorbing substrate, we show that only the contribution of the transmitted wave is insensitive to the direction of illumination, by analogy with the textbook case of transmission through a planar stratified medium. This property was recently confirmed experimentally [J. Phys. Chem. C 123, 15217 (2019)] and is established here on theoretical grounds in the frameworks of the Green's function integral equation method and the Lorentz reciprocity theorem. Some of its limitations with regard to the incidence and the polarization of the excitation wave are also discussed in detail.
\end{abstract}

DOI: 10.1103/PhysRevA.102.033521

\section{INTRODUCTION}

A particle embedded in a non-absorbing homogeneous dielectric medium (real refractive index $n$ ) and submitted to an electromagnetic plane wave excitation (intensity $I_{i}$, electric field vector amplitude $\mathbf{E}^{(i)}$, angular frequency $\omega=k_{0} c=\frac{2 \pi}{\lambda_{0}} c$, and wave vector $\mathbf{k}_{i}$ with $k_{i}=n k_{0}$ ) dissipates part of the incident power through elastic (Rayleigh) scattering into the whole space and through ohmic losses if it is absorbing. The extinguished power $P^{(\mathrm{ext})}$ is defined as the sum of the absorbed $\left(P^{(\mathrm{abs})}\right)$ and scattered $\left(P^{(\mathrm{scat})}\right)$ powers. The optical theorem states that the extinction cross section defined as the ratio $C^{(\mathrm{ext})}=P^{(\mathrm{ext})} / I_{i}$ is merely related to interferences between the excitation wave and the scattered wave, which asymptotically adopts a transverse spherical wave structure $\left[\mathbf{E}^{(s)}\left(\mathbf{k}_{\mathbf{i}}, \mathbf{r}\right)=\right.$ $\mathbf{A}(\hat{\mathbf{r}}=\mathbf{r} / r) e^{i k_{i} r} / r$ with $\left.\mathbf{A}(\hat{\mathbf{r}}=\mathbf{r} / r) \cdot \mathbf{r}=0\right][1,2]$. It can be expressed simply in the form $C^{(\mathrm{ext})}=\frac{2 \pi \varepsilon_{0} c}{k_{0} I_{i}} \operatorname{Im}\left[\mathbf{E}^{(i) *} \cdot \mathbf{A}\left(\hat{\mathbf{k}}_{\boldsymbol{i}}\right)\right]$ (SI units) as a simple function of the projection of the far-field scattering vector amplitude in the propagation direction of the incident wave $\left(\hat{\mathbf{r}}=\hat{\mathbf{k}}_{i}=\mathbf{k}_{\mathbf{i}} / k_{i}\right.$ ) onto the electric-field vector amplitude of this incident wave [Fig. 1(a)].

The optical theorem can be extended to more complex situations provided that the translational symmetry of the plane-wave excitation is retained, as in the case of a scatterer included in a stratified medium made of stacked plane parallel and nonabsorbing slabs [Fig. 1(b)]. It is then excited both by (i) the backward- and forward-propagating waves generated by the multiple reflections of the incident wave inside the layered medium and (ii) a part of the scattered field redirected back to it because of these reflections. Whatever the structure of the layered medium, the incident wave gives rise to transmitted and reflected plane waves in the far-field regions. Part of the scattered wave is confined and guided in the layered medium whereas the rest is emitted in the upper or the lower free half spaces. The generalized optical theorem states that the total extinction cross section can be split into two contributions $C^{(\mathrm{ext})}=C_{\mathrm{ref}}^{(\mathrm{ext})}+C_{\text {trans }}^{(\mathrm{ext})}$ with $C_{\mathrm{ref}}^{(\mathrm{ext})}=$ $\frac{2 \pi \varepsilon_{0} c}{k_{0} I_{i}} \operatorname{Im}\left[\mathbf{E}^{(r) *} \cdot \mathbf{A}\left(\hat{\mathbf{k}}_{\boldsymbol{r}}\right)\right]$ and $C_{\text {trans }}^{(\mathrm{ext})}=\frac{2 \pi \varepsilon_{0} c}{k_{0} I_{i}} \operatorname{Im}\left[\mathbf{E}^{(t) *} \cdot \mathbf{A}\left(\hat{\mathbf{k}}_{t}\right)\right]$, by analogy with the homogeneous-medium case [3-6]. These expressions now involve, respectively, the electric fields of the reflected and transmitted waves and the far fields scattered in their exact direction of propagation $\left(\hat{\mathbf{k}}_{r}\right.$ and $\left.\hat{\mathbf{k}}_{t}\right)$, labeled as backward (BW) and forward (FW) in Fig. 2(b).

The predictions of the generalized optical theorem were verified quantitatively and discussed in a recent experimental study on the optical extinction of single plasmonic particles supported on a transparent substrate (monolayer slab) [7]. On this occasion, we highlighted the general result that the extinction of the transmitted wave itself is unchanged whether the particle is located at the inlet or the outlet side of the slab [up or down configurations in Fig. 1(c)]. Considering a fixed position of the scatterer relative to the interface, this is equivalent to saying that the extinction of the transmitted wave remains the same, whether the scatterer is illuminated from the upper or from the lower medium or, in other terms, if the directions of the incident and transmitted waves are swapped.

Indeed, in the absence of an interface (scatterer in a homogeneous dielectric medium), this property can be inferred from the Lorentz principle of reciprocity in optics [8-16] that was comprehensively formulated by de Hoop in case of electromagnetic scattering by an isolated object of finite size [17-19]. If two plane waves with electric fields $\mathbf{E}^{1}$ and $\mathbf{E}^{2}$ are incident on a scatterer in the respective directions $\hat{\mathbf{s}}_{1}$ and $\hat{\mathbf{s}}_{\mathbf{2}}$, de Hoop established the reciprocal relation
$\mathbf{E}^{1} \cdot \mathbf{A}_{E^{2}}\left(-\hat{\mathbf{s}}_{1}\right)=\mathbf{E}^{2} \cdot \mathbf{A}_{\boldsymbol{E}^{1}}\left(-\hat{\mathbf{s}}_{2}\right)$, 

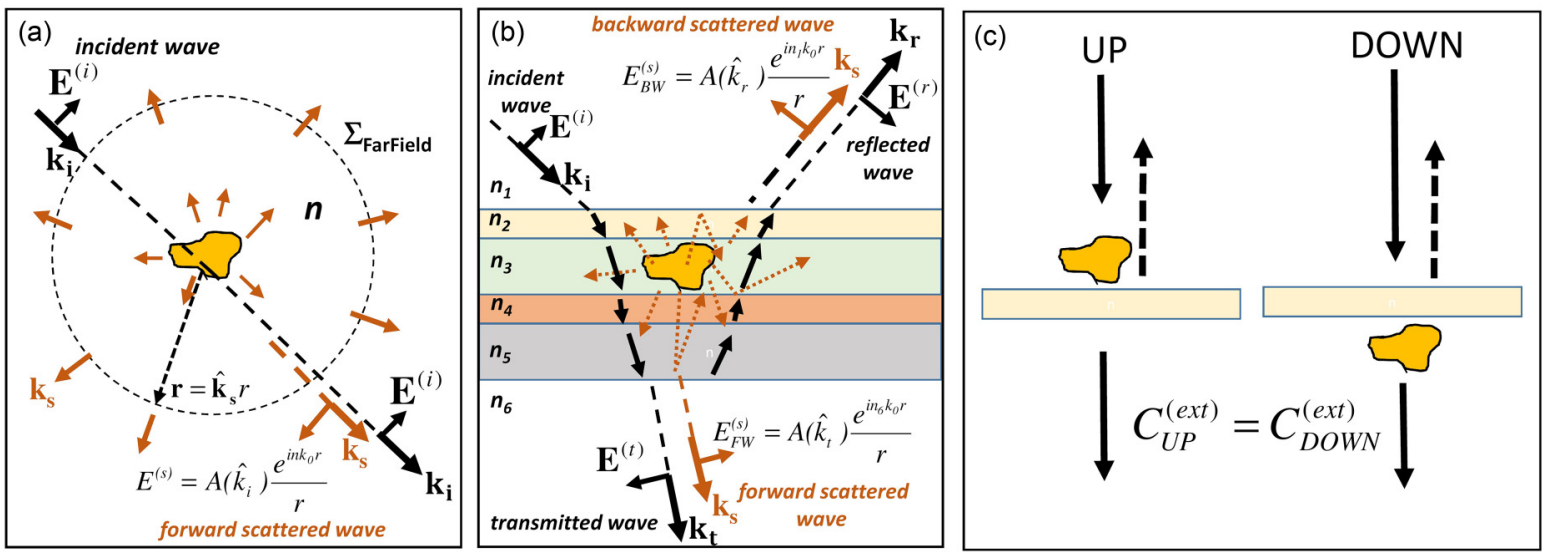

FIG. 1. Illustration of selected configurations that fall within the scope of the optical theorem. The scatterer is subject to a plane-wave excitation and can be embedded in (a) a homogeneous or (b) a stratified medium. (c) For a scatterer located near a dielectric slab, a previous experimental study showed that the extinction of the transmitted wave at normal incidence is independent of the direction of illumination [7].

where $\mathbf{A}_{\mathbf{E}^{i}}\left(-\hat{\mathbf{s}}_{\mathbf{j}}\right)$ is the vector amplitude of the far scattered field induced by wave $i$ (electric-field vector amplitude $\mathbf{E}^{i}$ ) that propagates, at large distances, in the direction opposite to the direction of wave $j$ (electric-field vector amplitude $\mathbf{E}^{j}$ ). In the very particular configuration of counter-propagating waves $\left(\hat{\mathbf{s}}_{1}=-\hat{\mathbf{s}}_{2}\right)$, this relation directly leads, through the optical theorem formulation, to the invariance of the extinction cross sections relative to a simple reversal of the directions of illumination $\left(\mathbf{E}^{1} \leftrightarrow \mathbf{E}^{2}\right)$. However, such an invariance is not straightforward in the case of a scatterer close to an interface between two dielectric media because of the splitting of the exciting waves among secondary refracted and reflected waves.

The aim of this study is to define and discuss the general conditions required to establish the invariance of the extinction cross sections in transmission relative to a permutation of the incident and transmitted wave directions, that is, when the illumination is turned from the upper to the lower medium, as was observed experimentally and proved in the frame of a simple model (small scatterer and normal incidence) [7].

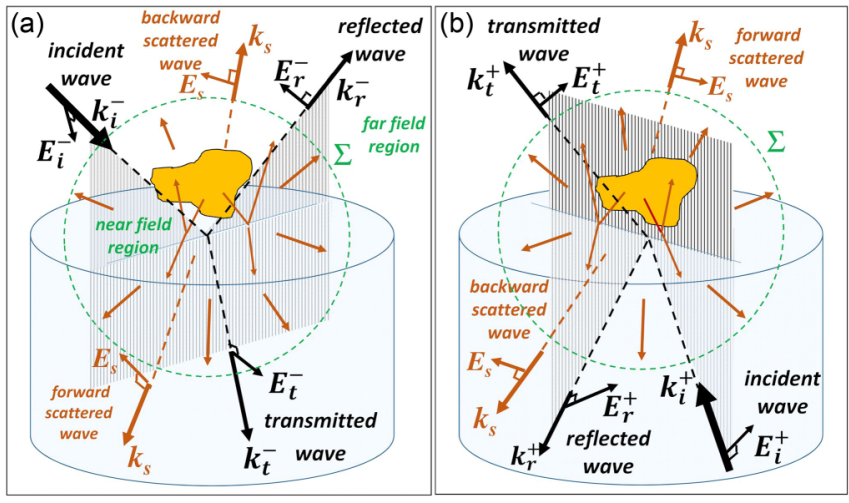

FIG. 2. General excitation schemes for a scatterer located above the interface between two dielectric media and irradiated by a plane wave coming (a) from the upper half space (air) or (b) from the lower half space (dielectric medium). A sphere of radius $\Sigma$ enclosing the particle is drawn to arbitrarily distinguish between near-field and farfield regions.
In Sec. II, we introduce general notations and the theoretical background for formulating the generalized optical theorem. It is based on the Green's function formalism and adapted from the reference paper of Lytle et al. [3] but using here the SI system of units. We consider the simplest configuration where a scatterer placed above an infinite planar interface between air and a dielectric medium is excited by a plane wave originating from either one or the other half space. This basic model can be straightforwardly extended to a plane-parallel dielectric slab of infinite lateral extension embedded in air. In Sec. III, we establish the conditions for ensuring the exact or approximate invariance of the optical extinction in transmission or reflection when reversing the direction of propagation of the incident wave. Two different approaches are used: a Green's function integral equation formalism and the direct application of general reciprocity principles expressed from the Lorentz theorem [20]. In Sec. IV, we discuss these properties in the case of an excitation at normal incidence, particularly their consequences with regard to the optical extinction of two-dimensional (2D) planar chiral objects, and we illustrate from numerical simulations their limitations in the more general case of an excitation at oblique incidence.

\section{DEFINITIONS AND NOTATIONS}

The system addressed here is illustrated in Fig. 2. The space is divided into two halves separated by a planar interface. A non-magnetic scatterer is kept positioned in the upper half space chosen as air for convenience (index of refraction $n_{1}=1$ ). The lower half space consists of a semi-infinite nonmagnetic and non-absorbing homogeneous medium (index of refraction $n_{2}=n$ ). The relative electric permittivity writes $\varepsilon(\mathbf{r}) / \varepsilon_{0}=n^{2}$ in the lower space and $\varepsilon(\mathbf{r}) / \varepsilon_{0}=1+\eta(\mathbf{r})$ in the upper space. The electric susceptibility $\eta(\mathbf{r})$ is zero except in the volume $V_{\mathrm{s}}$ of the scatterer. The relative magnetic permeability $\mu(\mathbf{r}) / \mu_{0}$ is equal to unity in all media, including the scatterer. In the following, the scatterer is supposed to be excited by transverse plane waves generated by distant sources located either in the upper [superscript ${ }^{(-)}$in Fig. 2(a)] or in the lower [superscript ${ }^{(+)}$in Fig. 2(b)] half space, with an arbitrary incidence relative to the surface normal ( $z$ axis). The 


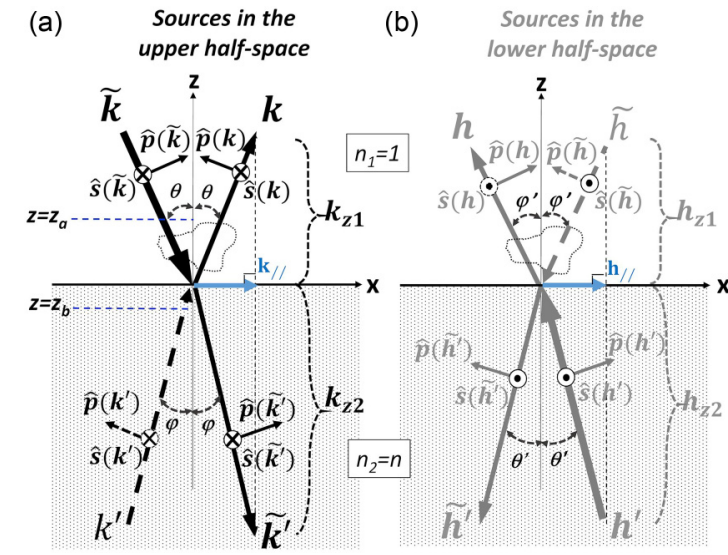

FIG. 3. Wave-vector notations, in the plane of incidence, for incident, reflected, and transmitted plane waves in the case of an excitation from the upper half space [black arrows in panel (a)] or from the lower half space [gray arrows in panel (b)]. The figure indicates the convention used for the orientation of the corresponding TE (s polarized) and TM (p polarized) basis vectors.

incident waves propagate along the negative or positive $z$ values for sources in the upper or lower half space, respectively. The wave vectors of the incident, reflected, and transmitted plane waves are denoted $\mathbf{k}_{i}^{ \pm}, \mathbf{k}_{r}^{ \pm}$, and $\mathbf{k}_{t}^{ \pm}$, in Fig. 2 and the incident electric field is $\mathbf{E}^{(i) \pm}\left(\mathbf{r}, \mathbf{k}_{i}^{ \pm}, t\right)=\operatorname{Re}\left[\mathbf{E}^{(i)}\left(\mathbf{r}, \mathbf{k}_{i}^{ \pm}\right) e^{-i \omega t}\right]$. In the following, the harmonic time-dependent factor $e^{-i \omega t}$ is omitted.

\section{A. The excitation fields}

Boundary conditions at the interface normal to the $z$ axis impose the conservation of in-plane $x$ and $y$ components (Snell-Descartes law). In the fixed basis $(\hat{\mathbf{x}}, \hat{\mathbf{y}}, \hat{\mathbf{z}})$, the wave vectors of the incident, reflected, and transmitted parts of the field produced by far sources located in the upper space are rather denoted $\mathbf{k}_{i}^{-}=\tilde{\mathbf{k}} \equiv\left(k_{x}, k_{y},-k_{z 1}\right), \mathbf{k}_{r}^{-}=\mathbf{k} \equiv$ $\left(k_{x}, k_{y}, k_{z 1}\right)$, and $\mathbf{k}_{t}^{-}=\tilde{\mathbf{k}}^{\prime} \equiv\left(k_{x}, k_{y},-k_{z 2}\right)$ and those of the field produced by far sources located in the lower space, $\mathbf{k}_{i}^{+}=\mathbf{h}^{\prime} \equiv\left(h_{x}, h_{y}, h_{z 2}\right), \mathbf{k}_{r}^{+}=\tilde{\mathbf{h}}^{\prime} \equiv\left(h_{x}, h_{y},-h_{z 2}\right)$ and $\mathbf{k}_{t}^{+}=$ $\mathbf{h} \equiv\left(h_{x}, h_{y}, h_{z 1}\right)$, so as to match with the notation of Ref. [3] (see Fig. 3). For completeness, additional wave vectors $\mathbf{k}^{\prime} \equiv$ $\left(k_{x}, k_{y}, k_{z 2}\right)$ and $\tilde{\mathbf{h}} \equiv\left(h_{x}, h_{y},-h_{z 1}\right)$ can also be introduced even if they are not directly involved in the respective excitation schemes. The prime index refers to the lower-half-space medium and the tilde character to a propagation towards negative $z$ values. Reflected and transmitted wave vectors are fully determined by the choice of the incident wave vectors and obey the relations

$$
\begin{aligned}
& \mathbf{K}_{\|}=\tilde{\mathbf{K}}_{\|}=\mathbf{K}_{\|}^{\prime}=\tilde{\mathbf{K}}_{\|}^{\prime}=K_{x} \hat{\mathbf{x}}+K_{y} \hat{\mathbf{y}}, \\
& \text { and } K_{z 1}=\left\{\begin{array}{l}
\sqrt{k_{0}^{2}-K_{\|}^{2}} \quad \text { when } k_{0}^{2}>K_{\|}^{2} \\
i \sqrt{-k_{0}^{2}+k_{\|}^{2}} \quad \text { when } k_{0}^{2}<K_{\|}^{2},
\end{array}\right. \\
& K_{z 2}=\left\{\begin{array}{l}
\sqrt{n^{2} k_{0}^{2}-K_{\|}^{2}} \quad \text { when } n^{2} k_{0}^{2}>K_{\|}^{2} \\
i \sqrt{-n^{2} k_{0}^{2}+K_{\|}^{2}} \quad \text { when } n^{2} k_{0}^{2}<K_{\|}^{2},
\end{array}\right.
\end{aligned}
$$

with $\mathbf{K}=\mathbf{k}$ or $\mathbf{K}=\mathbf{h}$.
The total electric fields generated by sources in the upper and in the lower half spaces are written, respectively $[\Theta(z)$ is the Heaviside step function],

$$
\begin{aligned}
\mathbf{E}^{(\mathrm{inc})-}(\tilde{\mathbf{k}}, \mathbf{r})= & \Theta(z)\left[\mathbf{E}^{(i)^{-}}(\tilde{\mathbf{k}}, \mathbf{r})+\mathbf{E}^{(r)^{-}}(\mathbf{k}, \mathbf{r})\right] \\
& +\Theta(-z) \mathbf{E}^{(t)^{-}}\left(\widetilde{\mathbf{k}^{\prime}}, \mathbf{r}\right)
\end{aligned}
$$

and

$$
\begin{aligned}
\mathbf{E}^{(\mathrm{inc})+}\left(\mathbf{h}^{\prime}, \mathbf{r}\right)= & \Theta(-z)\left[\mathbf{E}^{(i)^{+}}\left(\mathbf{h}^{\prime}, \mathbf{r}\right)+\mathrm{E}^{(r)^{+}}\left(\tilde{\mathbf{h}}^{\prime}, \mathbf{r}\right)\right] \\
& +\Theta(z) \mathbf{E}^{(t)^{+}}(\mathbf{h}, \mathbf{r})
\end{aligned}
$$

where the incident, reflected, and transmitted electric fields can be expressed in the form

$$
\begin{aligned}
\mathbf{E}^{(i)^{-}}(\tilde{\mathbf{k}}, \mathbf{r}) & =\mathbf{e}^{-}(\tilde{\mathbf{k}}) e^{i \tilde{\mathbf{k}} \cdot \mathbf{r}} \\
\mathbf{E}^{(r)^{-}}(\mathbf{k}, \mathbf{r}) & =\mathbf{e}^{(r)-}(\mathbf{k}) e^{i \mathbf{k} \cdot \mathbf{r}}, \\
\mathbf{E}^{(t)^{-}}\left(\tilde{\mathbf{k}^{\prime}}, \mathbf{r}\right) & =\mathbf{e}^{(t)-}\left(\tilde{\mathbf{k}}^{\prime}\right) e^{i \tilde{\mathbf{k}}^{\prime} \cdot \mathbf{r}}
\end{aligned}
$$

and

$$
\begin{aligned}
\mathbf{E}^{(i)+}\left(\mathbf{h}^{\prime}, \mathbf{r}\right) & =\mathbf{e}^{+}\left(\mathbf{h}^{\prime}\right) e^{i \mathbf{h}^{\prime} \cdot \mathbf{r}} \\
\mathbf{E}^{(r)+}\left(\tilde{\mathbf{h}}^{\prime}, \mathbf{r}\right) & =\mathbf{e}^{(r)+}\left(\tilde{\mathbf{h}}^{\prime}\right) e^{i \tilde{\mathbf{h}}^{\prime} \cdot \mathbf{r}}, \\
\mathbf{E}^{(t)+}(\mathbf{h}, \mathbf{r}) & =\mathbf{e}^{(t)+}(\mathbf{h}) e^{i \mathbf{h} \cdot \mathbf{r}}
\end{aligned}
$$

Reflected and transmitted electric-field amplitudes are fully determined by boundary conditions at the interface through the action of reflection and transmission operators

$$
\begin{aligned}
\mathbf{e}^{(r)-}(\mathbf{k}) & =\hat{R}(\mathbf{k}, \tilde{\mathbf{k}}) \mathbf{e}^{-}(\tilde{\mathbf{k}}), \\
\mathbf{e}^{(r)+}\left(\tilde{\mathbf{h}}^{\prime}\right) & =\hat{R}^{\prime}\left(\tilde{\mathbf{h}}^{\prime}, \mathbf{h}^{\prime}\right) \mathbf{e}^{+}\left(\mathbf{h}^{\prime}\right), \\
\mathbf{e}^{(t)-}\left(\tilde{\mathbf{k}}^{\prime}\right) & =\hat{T}\left(\tilde{\mathbf{k}}^{\prime}, \tilde{\mathbf{k}}\right) \mathbf{e}^{-}(\tilde{\mathbf{k}}), \\
\text { and } \quad \mathbf{e}^{(t)+}(\mathbf{h}) & =\hat{T}^{\prime}\left(\mathbf{h}, \mathbf{h}^{\prime}\right) \mathbf{e}^{+}\left(\mathbf{h}^{\prime}\right) .
\end{aligned}
$$

For an oblique incidence and in order to apply the Fresnel laws for reflection and transmission, it is necessary to distinguish transverse electric (TE) from transverse magnetic (TM) polarized waves. The transverse electric field $\mathbf{e}(\mathbf{K})$ of a plane wave with wave number $\mathbf{K} \equiv\left(K_{x}, K_{y}, K_{z}\right)$ (unit vector $\hat{\mathbf{K}}=\mathbf{K} /|\mathbf{K}|)$ can be expressed in the TE-TM basis:

where

$$
\mathbf{e}(\mathbf{K})=e_{s}(\mathbf{K}) \hat{\boldsymbol{s}}(\mathbf{K})+e_{p}(\mathbf{K}) \hat{\boldsymbol{p}}(\mathbf{K}),
$$

$$
\hat{\boldsymbol{s}}(\mathbf{K})=\frac{1}{|\hat{\mathbf{z}} \times \hat{\mathbf{K}}|} \hat{\mathbf{z}} \times \hat{\mathbf{K}}=\frac{1}{\sqrt{\mathbf{K}_{\|}^{2}}}\left(-K_{y}, K_{x}, 0\right)
$$

and

$$
\begin{aligned}
\hat{\boldsymbol{p}}(\mathbf{K}) & =-\frac{1}{|\hat{\boldsymbol{s}}(\mathbf{K}) \times \hat{\mathbf{K}}|} \hat{\boldsymbol{s}}(\mathbf{K}) \times \hat{\mathbf{K}} \\
& =\frac{1}{K_{0} \sqrt{K_{\|}^{2}}}\left(-K_{x} K_{z},-K_{y} K_{z}, K_{\|}^{2}\right) .
\end{aligned}
$$

$\hat{\boldsymbol{p}}(\mathbf{K})$ and $\hat{\boldsymbol{s}}(\mathbf{K})$ are real unit vectors, respectively parallel and normal to the plane of incidence so that $(\hat{\boldsymbol{s}}(\mathbf{K}), \hat{\boldsymbol{p}}(\mathbf{K}), \hat{\mathbf{K}})$ forms 
a direct and orthogonal basis set [21]. They are represented in Fig. 3 for all the wave vectors considered here.

The reflection and transmission operators can be developed as dyadics to more clearly individualize their action on TE [polarization along $\hat{\boldsymbol{s}}(\mathbf{K})$ ] or TM [polarization along $\hat{\boldsymbol{p}}(\mathbf{K})$ ] components:

$$
\begin{gathered}
\hat{R}(\mathbf{k}, \tilde{\mathbf{k}})=r_{s}\left(k_{\|}\right) \hat{\boldsymbol{s}}(\mathbf{k}) \otimes \hat{\boldsymbol{s}}(\tilde{\mathbf{k}})+r_{p}\left(k_{\|}\right) \hat{\boldsymbol{p}}(\mathbf{k}) \otimes \hat{\boldsymbol{p}}(\tilde{\mathbf{k}}), \\
\hat{R}^{\prime}\left(\tilde{\mathbf{h}}^{\prime}, \mathbf{h}^{\prime}\right)=r_{s}^{\prime}\left(h_{\|}\right) \hat{\boldsymbol{s}}\left(\tilde{\mathbf{h}}^{\prime}\right) \otimes \hat{\boldsymbol{s}}\left(\mathbf{h}^{\prime}\right)+r_{p}^{\prime}\left(h_{\|}\right) \hat{\boldsymbol{p}}\left(\tilde{\mathbf{h}}^{\prime}\right) \otimes \hat{\boldsymbol{p}}\left(\mathbf{h}^{\prime}\right), \\
\hat{T}\left(\tilde{\mathbf{k}}^{\prime}, \tilde{\mathbf{k}}\right)=t_{s}\left(k_{\|}\right) \hat{\boldsymbol{s}}\left(\tilde{\mathbf{k}}^{\prime}\right) \otimes \hat{\boldsymbol{s}}(\tilde{\mathbf{k}})+t_{p}\left(k_{\|}\right) \hat{\boldsymbol{p}}\left(\tilde{\mathbf{k}}^{\prime}\right) \otimes \hat{\boldsymbol{p}}(\tilde{\mathbf{k}}), \\
\hat{T}^{\prime}\left(\mathbf{h}, \mathbf{h}^{\prime}\right)=t_{s}^{\prime}\left(h_{\|}\right) \hat{\boldsymbol{s}}(\mathbf{h}) \otimes \hat{\boldsymbol{s}}\left(\mathbf{h}^{\prime}\right)+t_{p}^{\prime}\left(h_{\|}\right) \hat{\boldsymbol{p}}(\mathbf{h}) \otimes \hat{\boldsymbol{p}}\left(\mathbf{h}^{\prime}\right),
\end{gathered}
$$

where $r_{(s / p)}\left(k_{\|}\right), \quad r_{(s / p)}^{\prime}\left(h_{\|}\right), \quad t_{(s / p)}\left(k_{\|}\right)$, and $t_{(s / p)}^{\prime}\left(h_{\|}\right)$are the Fresnel coefficients for reflection and transmission of up-coming or down-coming TE and TM plane waves (Appendix A). The prime symbol refers to an incident wave coming from the dielectric medium (sources in the lower half space). The vector amplitudes of the corresponding reflected and transmitted waves in Eqs. (4a) and (4b) are fully determined as a function of the coefficients of the incident fields through the relations

$$
\begin{aligned}
& \mathbf{e}^{(r)-}(\mathbf{k})=\hat{R}(\mathbf{k}, \tilde{\mathbf{k}}) \mathbf{e}^{-}(\tilde{\mathbf{k}}) \\
& =r_{s}\left(k_{\|}\right) e_{s}^{-}(\tilde{\mathbf{k}}) \hat{\boldsymbol{s}}(\mathbf{k})+r_{p}\left(k_{\|}\right) e_{p}^{-}(\tilde{\mathbf{k}}) \hat{\boldsymbol{p}}(\mathbf{k}), \\
& \mathbf{e}^{(t)-}\left(\tilde{\mathbf{k}}^{\prime}\right)=\hat{T}\left(\tilde{\mathbf{k}}^{\prime}, \tilde{\mathbf{k}}\right) \mathbf{e}^{-}(\tilde{\mathbf{k}})
\end{aligned}
$$

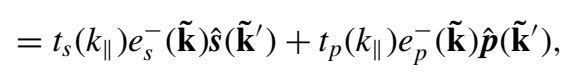

$$
\begin{aligned}
& \mathbf{e}^{(r)+}\left(\tilde{\mathbf{h}}^{\prime}\right)=\hat{R}^{\prime}\left(\tilde{\mathbf{h}}^{\prime}, \mathbf{h}^{\prime}\right) \mathbf{e}^{+}\left(\mathbf{h}^{\prime}\right) \\
& =r_{s}^{\prime}\left(h_{\|}\right) e_{s}^{+}\left(\mathbf{h}^{\prime}\right) \hat{\boldsymbol{s}}\left(\tilde{\mathbf{h}}^{\prime}\right)+r_{p}^{\prime}\left(h_{\|}\right) e_{p}^{+}\left(\mathbf{h}^{\prime}\right) \hat{\boldsymbol{p}}\left(\tilde{\mathbf{h}}^{\prime}\right), \\
& \mathbf{e}^{(t)+}(\mathbf{h})=\hat{T}^{\prime}\left(\mathbf{h}, \mathbf{h}^{\prime}\right) \mathbf{e}^{+}\left(\mathbf{h}^{\prime}\right) \\
& =t_{s}^{\prime}\left(h_{\|}\right) e_{s}^{+}\left(\mathbf{h}^{\prime}\right) \hat{\boldsymbol{s}}(\mathbf{h})+t_{p}^{\prime}\left(h_{\|}\right) e_{p}^{+}\left(\mathbf{h}^{\prime}\right) \hat{\boldsymbol{p}}(\mathbf{h}),
\end{aligned}
$$

where $e_{(s / p)}^{-}(\tilde{\mathbf{k}})$ and $e_{(s / p)}^{+}\left(\mathbf{h}^{\prime}\right)$ are the components of the incident electric field vectors $\mathbf{e}^{-}(\tilde{\mathbf{k}})$ and $\mathbf{e}^{+}\left(\mathbf{h}^{\prime}\right)$ on their respective TE-TM basis [see Eq. (5)].

\section{B. The scattered fields in the Green's function formalism}

The total electric field is the sum of the incident and the scattered fields,

$$
\mathbf{E}^{(\text {tot })}(\mathbf{K}, \mathbf{r})=\mathbf{E}^{(\text {inc })}(\mathbf{K}, \mathbf{r})+\mathbf{E}^{(\text {scat })}(\mathbf{K}, \mathbf{r}),
$$

with $\mathbf{K}=\tilde{\mathbf{k}}$ or $\mathbf{h}^{\prime}$, depending on the configuration. In the framework of the Green's function formalism [3,18,21,22] the scattered field is solution of the integral equation

$$
\begin{aligned}
\mathbf{E}^{(\text {scat })}(\mathbf{K}, \mathbf{r})= & k_{0}^{2} \iiint_{\mathrm{Vs}} d^{3} r^{\prime} \eta\left(\mathbf{r}^{\prime}\right) \hat{G}\left(\mathbf{r}, \mathbf{r}^{\prime}\right) \\
& \times\left[\mathbf{E}^{\text {(inc) }}\left(\mathbf{K}, \mathbf{r}^{\prime}\right)+\mathbf{E}^{\text {(scat })}\left(\mathbf{K}, \mathbf{r}^{\prime}\right)\right]
\end{aligned}
$$

where $\hat{G}\left(\mathbf{r}, \mathbf{r}^{\prime}\right)$ is the Green operator suited to the present problem. It can be expressed as a plane-wave expansion (angular spectral representation or Weyl identity [21]) in the form

$$
\begin{aligned}
\hat{G}\left(\mathbf{r}, \mathbf{r}^{\prime}\right)= & \frac{i}{8 \pi^{2}} \iint_{K_{x}, K_{y}} \frac{d^{2} K_{\|}}{K_{z}} e^{i \mathbf{K}_{\|} \cdot\left(\mathbf{r}_{\|}-\mathbf{r}_{\|}^{\prime}\right)} \\
& \times\left\{\Theta(-z) \hat{T}\left(\tilde{\mathbf{K}}^{\prime}, \tilde{\mathbf{K}}\right) e^{i\left(K_{z} \mathbf{z}^{\prime}-\mathbf{K}_{z}^{\prime} z\right)}\right.
\end{aligned}
$$

$$
\begin{aligned}
& +\Theta(z)\left[\hat{R}(\mathbf{K}, \tilde{\mathbf{K}}) e^{i K_{z}\left(\mathrm{z}+z^{\prime}\right)}+\Theta\left(z-z^{\prime}\right) \hat{I}(\mathbf{K}, \mathbf{K})\right. \\
& \left.\left.\times e^{i K_{z}\left(\mathrm{z}-z^{\prime}\right)}+\Theta\left(z^{\prime}-z\right) \hat{I}(\tilde{\mathbf{K}}, \tilde{\mathbf{K}}) e^{i K_{z}\left(\mathrm{z}^{\prime}-z\right)}\right]\right\},
\end{aligned}
$$

considering that the scatterer remains located in the upperhalf space $\left(z^{\prime}>0\right)[3,4,21,23-25]$. The integration runs over all possible values of $K_{x}$ and $K_{y}$ from $-\infty$ to $+\infty\left(\mathbf{K}_{\|}=\right.$ $\left.K_{x} \hat{\boldsymbol{x}}+K_{y} \hat{\boldsymbol{y}}\right)$. The wave vectors $\mathbf{K}, \widetilde{\mathbf{K}}$, and their partners $\tilde{\mathbf{K}}^{\prime}$ and $\mathbf{K}^{\prime}$ are related through the application of Snell-Descartes laws at the interface: $\mathbf{K}=\mathbf{K}_{\|}+K_{z} \hat{z}, \tilde{\mathbf{K}}=\mathbf{K}_{\|}-K_{z} \hat{z}, \mathbf{K}^{\prime}=$ $\mathbf{K}_{\|}+K_{z}^{\prime} \hat{z}$, and $\tilde{\mathbf{K}}^{\prime}=\mathbf{K}_{\|}-K_{z}^{\prime} \hat{\boldsymbol{z}}$, with $K_{z}$ and $K_{z}^{\prime}$ defined according to the same type of relations as those established for excitation fields in Eq. (2). Using suitable TE-TM basis sets, the operators $\hat{T}\left(\widetilde{\mathbf{K}}^{\prime}, \tilde{\mathbf{K}}\right)$ and $\hat{R}(\mathbf{K}, \tilde{\mathbf{K}})$ are constructed as the dyadics defined in Eq. (8). In Eq. (12), $\hat{I}(\mathbf{K}, \mathbf{K})=\hat{1}-\hat{\mathbf{K}} \otimes$ $\hat{\mathbf{K}}=\hat{\boldsymbol{s}}(\mathbf{K}) \otimes \hat{\boldsymbol{s}}(\mathbf{K})+\hat{\boldsymbol{p}}(\mathbf{K}) \otimes \hat{\boldsymbol{p}}(\mathbf{K})$ is the projector onto the plane normal to $\mathbf{K}(\hat{1}$ is the identity operator). For a transverse wave $\left[\mathbf{e}(\mathbf{K})=e_{s}(\mathbf{K}) \hat{\boldsymbol{s}}(\mathbf{K})+e_{p}(\mathbf{K}) \hat{\boldsymbol{p}}(\mathbf{K})\right], \hat{I}(\mathbf{K}, \mathbf{K})$ reduces to the identity operator $[\hat{I}(\mathbf{K}, \mathbf{K}) \mathbf{e}(\mathbf{K})=\mathbf{e}(\mathbf{K})]$.

As can be seen in Fig. 2, the scattered field depends on the excitation configuration $(+/-)$ through $\mathbf{E}^{(\text {inc })}(\mathbf{K}, \mathbf{r})$ defined by Eqs. (3) and (4) and also on the half space into which it propagates $(z<0$ or $z>0)$. The scattered fields corresponding to the four possible cases behave asymptotically as transverse spherical waves in the far zone $\left(r k_{0} \gg 1\right)$, with the following angular spectrum representations:

$$
\begin{aligned}
& \mathbf{E}^{\text {(scat) }-}(\tilde{\mathbf{k}}, \mathbf{r}, \mathrm{z}>0)=\frac{i}{8 \pi^{2}} \iint \frac{d \mathrm{~K}_{x} d \mathrm{~K}_{y}}{K_{z}} \mathbf{A}_{\uparrow}^{-}(\mathbf{K}, \tilde{\mathbf{k}}) \\
& \times e^{i \mathrm{~K} \cdot \mathbf{r}} \underset{r \rightarrow \infty}{\longrightarrow} \mathbf{A}_{\uparrow}^{-}\left(k_{0} \frac{\mathbf{r}}{r}, \tilde{\mathbf{k}}\right) \frac{e^{i k_{0} r}}{r}, \\
& \mathbf{E}^{\text {(scat })-}(\tilde{\mathbf{k}}, \mathbf{r}, \mathrm{z}<0)=\frac{i}{8 \pi^{2}} \iint \frac{d \mathrm{~K}_{x} d \mathrm{~K}_{y}}{K^{\prime}{ }_{z}} \mathbf{A}_{\downarrow}^{-}\left(\tilde{\mathbf{K}}^{\prime}, \tilde{\mathbf{k}}\right) \\
& \times e^{i \tilde{\mathbf{K}}^{\prime} \cdot \mathbf{r}} \underset{r \rightarrow \infty}{\longrightarrow} \mathbf{A}_{\downarrow}^{-}\left(n k_{0} \frac{\mathbf{r}}{r}, \tilde{\mathbf{k}}\right) \frac{e^{i n k_{0} r}}{r}, \\
& \mathbf{E}^{\text {(scat })+}\left(\mathbf{h}^{\prime}, \mathbf{r}, \mathrm{z}>0\right)=\frac{i}{8 \pi^{2}} \iint \frac{d \mathrm{~K}_{x} d \mathrm{~K}_{y}}{K_{z}} \mathbf{A}_{\uparrow}^{+}\left(\mathbf{K}, \mathbf{h}^{\prime}\right) \\
& \times e^{i \mathrm{~K} \cdot \mathbf{r}} \underset{r \rightarrow \infty}{\longrightarrow} \mathbf{A}_{\uparrow}^{+}\left(k_{0} \frac{\mathbf{r}}{r}, \mathbf{h}^{\prime}\right) \frac{e^{i k_{0} r}}{r}, \\
& \mathbf{E}^{\text {(scat })+}\left(\mathbf{h}^{\prime}, \mathbf{r}, \mathrm{z}<0\right)=\frac{i}{8 \pi^{2}} \iint \frac{d \mathrm{~K}_{x} d \mathrm{~K}_{y}}{K_{z}^{\prime}} \mathbf{A}_{\downarrow}^{+}\left(\tilde{\mathbf{K}}^{\prime}, \mathbf{h}^{\prime}\right) \\
& \times e^{i \tilde{\mathbf{K}}^{\prime} \cdot \mathbf{r}} \underset{r \rightarrow \infty}{\longrightarrow} \mathbf{A}_{\downarrow}^{+}\left(n k_{0} \frac{\mathbf{r}}{r}, \mathbf{h}^{\prime}\right) \frac{e^{i n k_{0} r}}{r} .
\end{aligned}
$$

The far-field behaviors can be directly obtained through the application of the stationary phase method. In the following, the "个" and " $\downarrow$ " symbols will refer to $z>0$ and $z<0$ half spaces, respectively. The Fourier components $\mathbf{A}\left(\mathrm{k}_{\text {out }}, \mathbf{k}_{\text {in }}\right)$ are the amplitudes of the transverse far fields scattered with outcoming wave vector $\mathbf{k}_{\text {out }}$ after excitation by a plane wave with wave vector $\mathbf{k}_{\text {in }}$. Using Eqs. (11) and (12), the components $\mathbf{A}$ are expressed from the Fourier inversion of Eqs. (13) as integrals over the scatterer volume in the 
form

$$
\begin{aligned}
\mathbf{A}_{\downarrow}^{-}\left(\tilde{\mathbf{K}}^{\prime}, \tilde{\mathbf{k}}\right)= & \frac{K_{z}^{\prime}}{K_{z}} k_{0}^{2} \iiint_{V_{S}} d^{3} r \eta(\mathbf{r}) \hat{T}\left(\tilde{\mathbf{K}}^{\prime}, \tilde{\mathbf{K}}\right) \mathbf{E}^{(\mathrm{tot})-}(\tilde{\mathbf{k}}, \mathbf{r}) \\
& \times e^{-i \tilde{\mathbf{K}} \cdot \mathbf{r}}, \\
\mathbf{A}_{\uparrow}^{-}(\mathbf{K}, \tilde{\mathbf{k}})= & k_{0}^{2} \iiint_{V_{S}} d^{3} r \eta(\mathbf{r})\left[\hat{R}(\mathbf{K}, \tilde{\mathbf{K}}) \mathbf{E}^{(\mathrm{tot})-}(\tilde{\mathbf{k}}, \mathbf{r}) e^{-i \tilde{\mathbf{K}} \cdot \mathbf{r}}\right. \\
& \left.+\hat{I}(\mathbf{K}, \mathbf{K}) \mathbf{E}^{(\mathrm{tot})-}(\tilde{\mathbf{k}}, \mathbf{r}) e^{-i \mathbf{K} \cdot \mathbf{r}}\right], \\
\mathbf{A}_{\downarrow}^{+}\left(\tilde{\mathbf{K}}^{\prime}, \mathbf{h}^{\prime}\right)= & \frac{K_{z}^{\prime}}{K_{z}} k_{0}^{2} \iiint_{V_{S}} d^{3} r \eta(\mathbf{r}) \hat{T}\left(\tilde{\mathbf{K}}^{\prime}, \tilde{\mathbf{K}}\right) \mathbf{E}^{(\mathrm{tot})+}\left(\mathbf{h}^{\prime}, \mathbf{r}\right) \\
& \times e^{-i \tilde{\mathbf{K}} \cdot \mathbf{r}}, \\
\mathbf{A}_{\uparrow}^{+}\left(\mathbf{K}, \mathbf{h}^{\prime}\right)= & k_{0}^{2} \iiint_{V_{S}} d^{3} r \eta(\mathbf{r})\left[\hat{R}(\mathbf{K}, \tilde{\mathbf{K}}) \mathbf{E}^{(\mathrm{tot})+}\left(\mathbf{h}^{\prime}, \mathbf{r}\right) e^{-i \tilde{\mathbf{K}} \cdot \mathbf{r}}\right. \\
& \left.+\hat{I}(\mathbf{K}, \mathbf{K}) \mathbf{E}^{(\mathrm{tot})+}\left(\mathbf{h}^{\prime}, \mathbf{r}\right) e^{-i \mathbf{K} \cdot \mathbf{r}}\right] . \quad(14 \mathrm{~d})
\end{aligned}
$$

In each individual equation (14a)-(14d), the wave vectors $\mathbf{K}, \tilde{\mathbf{K}}, \tilde{\mathbf{K}}^{\prime}$, and $\mathbf{K}^{\prime}$ (the prime index and the tilde character have been defined in Sec. IIA) are related according to the Snell Descartes laws, depending on the process involved (transmission $T$ or reflection $R$ ). A derivation of Eq. (14a) is provided in Appendix B.

\section{Scattering operators in the Green's function integral formalism}

In the following, it will be useful to introduce the scattering operators that formally relate the vector amplitudes of the scattered fields to the amplitude of the incident fields according to

$$
\begin{aligned}
\mathbf{A}_{\downarrow}^{-}\left(\tilde{\mathbf{K}}^{\prime}, \tilde{\mathbf{k}}\right) & =\hat{\mathbf{S}}_{\downarrow}^{-}\left(\tilde{\mathbf{K}}^{\prime}, \tilde{\mathbf{k}}\right) \mathbf{e}^{-}(\tilde{\mathbf{k}}), \\
\mathbf{A}_{\uparrow}^{-}(\mathbf{K}, \tilde{\mathbf{k}}) & =\hat{\mathbf{S}}_{\uparrow}^{-}(\mathbf{K}, \tilde{\mathbf{k}}) \mathbf{e}^{-}(\tilde{\mathbf{k}}), \\
\mathbf{A}_{\downarrow}^{+}\left(\tilde{\mathbf{K}}^{\prime}, \mathbf{h}^{\prime}\right) & =\hat{\mathbf{S}}_{\downarrow}^{+}\left(\tilde{\mathbf{K}}^{\prime}, \mathbf{h}^{\prime}\right) \mathbf{e}^{+}\left(\mathbf{h}^{\prime}\right), \\
\mathbf{A}_{\uparrow}^{+}\left(\mathbf{K}, \mathbf{h}^{\prime}\right) & =\hat{\mathbf{S}}_{\uparrow}^{+}\left(\mathbf{K}, \mathbf{h}^{\prime}\right) \mathbf{e}^{+}\left(\mathbf{h}^{\prime}\right) .
\end{aligned}
$$

A difficulty is that the expressions of the vector amplitudes of the scattered fields in Eqs. (14) do not only depend explicitly on the incident field but also on the total field experienced by any point of the scatterer [see Eq. (10)]. In order to formally express the scattered fields and therefore the total fields as a function of the incident field, we assume that $\mathbf{E}^{\text {(scat) }}(\mathbf{K}, \mathbf{r})$ (with $\mathbf{K}=\tilde{\mathbf{k}}$ or $\mathbf{h}^{\prime}$ ) can be obtained from an iterative resolution of the implicit constitutive relation (11) and can be developed as the infinite converging series

$$
\mathbf{E}^{(\text {scat })}(\mathbf{K}, \mathbf{r})=k_{0}^{2} \sum_{i=0}^{\infty} \iiint_{\mathrm{Vs}} d^{3} r_{i} \eta\left(\mathbf{r}_{i}\right) \hat{\mathcal{G}}^{i}\left(\mathbf{r}, \mathbf{r}_{i}\right) \mathbf{E}^{(\text {inc })}\left(\mathbf{K}, \mathbf{r}_{i}\right),
$$

introducing the composed Green's operators of order $i$ (dimension $[\mathrm{L}]^{-1}$ ) defined as

$$
\hat{\mathcal{G}}^{0}\left(\mathbf{r}, \mathbf{r}_{0}\right)=\hat{G}\left(\mathbf{r}, \mathbf{r}_{0}\right)
$$

and

$$
\begin{aligned}
\hat{\mathcal{G}}^{i}\left(\mathbf{r}, \mathbf{r}_{i \geqslant 1}\right)= & k_{0}^{2} \iiint_{\mathrm{Vs}_{\mathrm{s}}} d^{3} r_{i-1} \eta\left(\mathbf{r}_{i-1}\right) \hat{\mathcal{G}}^{i}\left(\mathbf{r}, \mathbf{r}_{i-1}\right) \hat{G}\left(\mathbf{r}_{i-1}, \mathbf{r}_{i}\right) \\
= & k_{0}^{2 i} \iiint_{\mathrm{Vs}} d^{3} r_{0} \iiint_{\mathrm{Vs}} d^{3} r_{1} \cdots \iiint_{\mathrm{Vs}} d^{3} r_{i-1} \\
& \hat{G}\left(\mathbf{r}, \mathbf{r}_{0}\right) \eta\left(\mathbf{r}_{0}\right) \hat{G}\left(\mathbf{r}_{0}, \mathbf{r}_{1}\right) \eta\left(\mathbf{r}_{1}\right) \hat{G}\left(\mathbf{r}_{1}, \mathbf{r}_{2}\right) \eta\left(\mathbf{r}_{2}\right) \cdots \\
& \hat{G}\left(\mathbf{r}_{i-1}, \mathbf{r}_{i}\right) .
\end{aligned}
$$

These expressions are not rigorously valid because of the singularity of the Green's operator $\hat{G}\left(\mathbf{r}, \mathbf{r}^{\prime}\right)$ when $\mathbf{r}=\mathbf{r}^{\prime}$. Such a singularity is widely discussed in the literature [26-28] and can be overcome by a suitable modification detailed in Appendix C. As far as the explicit form of $\hat{G}\left(\mathbf{r}, \mathbf{r}^{\prime}\right)$ is not required in the following discussion, this modification that would make the notations more complicated is not of basic importance since the corrected function still possesses the suited analytical properties. For convenience, it will be disregarded in the following, without questioning the forthcoming conclusions and we will assume that both $\mathbf{E}^{\text {(scat })-}(\tilde{\mathbf{k}}, \mathbf{r})$ and $\mathbf{E}^{\text {(scat)+ }}\left(\mathbf{h}^{\prime}, \mathbf{r}\right)$ can be appropriately replaced by such an infinite converging series. Introducing the operator (dimension $[\mathrm{L}]$ ),

$$
\begin{aligned}
\hat{\Phi}\left(\mathbf{k}_{2}, \mathbf{k}_{1}\right)= & k_{0}^{2} \iiint_{V_{S}} d^{3} r \eta(\mathbf{r}) e^{i\left(\mathbf{k}_{1}-\mathbf{k}_{2}\right) \cdot \mathbf{r}} \hat{1}+k_{0}^{4} \sum_{i=0}^{\infty} \iiint_{V_{S}} d^{3} r \\
& \times \iiint_{V_{S}} d^{3} r_{i} \eta(\mathbf{r}) \eta\left(\mathbf{r}_{i}\right) e^{i\left(\mathbf{k}_{1} \cdot \mathbf{r}_{i}-\mathbf{k}_{2} \cdot \mathbf{r}\right)} \hat{\mathcal{G}}^{i}\left(\mathbf{r}, \mathbf{r}_{i}\right),
\end{aligned}
$$

it is possible, from the definition of the scattering vectors amplitude [Eqs. (14)] with the incident and scattered fields in Eqs. (4) and (16), to establish the simplified form of the scattering operators:

$$
\begin{aligned}
\hat{S}_{\downarrow}^{-}\left(\tilde{\mathbf{K}}^{\prime}, \tilde{\mathbf{k}}\right)= & \frac{k_{z 2}}{k_{z 1}}\left[\hat{T}\left(\tilde{\mathbf{K}}^{\prime}, \tilde{\mathbf{K}}\right) \hat{\Phi}(\tilde{\mathbf{K}}, \tilde{\mathbf{k}}) \hat{I}(\tilde{\mathbf{k}}, \tilde{\mathbf{k}})\right. \\
& \left.+\hat{T}\left(\tilde{\mathbf{K}}^{\prime}, \tilde{\mathbf{K}}\right) \hat{\Phi}(\tilde{\mathbf{K}}, \mathbf{k}) \hat{R}(\mathbf{k}, \tilde{\mathbf{k}})\right], \\
\hat{S}_{\uparrow}^{+}\left(\mathbf{K}, \mathbf{h}^{\prime}\right)= & {\left[\hat{R}(\mathbf{K}, \tilde{\mathbf{K}}) \hat{\Phi}(\tilde{\mathbf{K}}, \mathbf{h}) \widehat{T^{\prime}}\left(\mathbf{h}, \mathbf{h}^{\prime}\right)\right.} \\
& \left.+\hat{I}(\mathbf{K}, \mathbf{K}) \hat{\Phi}(\mathbf{K}, \mathbf{h}) \widehat{T^{\prime}}\left(\mathbf{h}, \mathbf{h}^{\prime}\right)\right], \\
\hat{S}_{\uparrow}^{-}(\mathbf{K}, \tilde{\mathbf{k}})= & {[\hat{R}(\mathbf{K}, \tilde{\mathbf{K}}) \hat{\Phi}(\tilde{\mathbf{K}}, \tilde{\mathbf{k}}) \hat{I}(\tilde{\mathbf{k}}, \tilde{\mathbf{k}})} \\
& +\hat{R}(\mathbf{K}, \tilde{\mathbf{K}}) \hat{\Phi}(\tilde{\mathbf{K}}, \mathbf{k}) \hat{R}(\mathbf{k}, \tilde{\mathbf{k}}) \\
& +\hat{I}(\mathbf{K}, \mathbf{K}) \hat{\Phi}(\mathbf{K}, \tilde{\mathbf{k}}) \hat{I}(\tilde{\mathbf{k}}, \tilde{\mathbf{k}}) \\
& +\hat{I}(\mathbf{K}, \mathbf{K}) \hat{\Phi}(\mathbf{K}, \mathbf{k}) \hat{R}(\mathbf{k}, \tilde{\mathbf{k}})], \\
\hat{S}_{\downarrow}^{+}\left(\tilde{\mathbf{K}}^{\prime}, \mathbf{h}^{\prime}\right)= & \frac{k_{z 2}}{k_{z 1}}\left[\hat{T}\left(\tilde{\mathbf{K}}^{\prime}, \tilde{\mathbf{K}}\right) \hat{\Phi}(\tilde{\mathbf{K}}, \mathbf{h}) \widehat{T^{\prime}}\left(\mathbf{h}, \mathbf{h}^{\prime}\right)\right] .
\end{aligned}
$$

In the expression of a scattering operator $\hat{S}\left(\mathbf{k}_{\text {out }}, \mathbf{k}_{\mathbf{i n}}\right)$, where $\mathbf{k}_{\mathbf{i n}}$ and $\mathbf{k}_{\text {out }}$ are the wave vectors of the incident and scattered waves wave, each generic term of the type $\widehat{W}\left(\mathbf{k}_{\text {out }}, \mathbf{u}\right) \hat{\Phi}(\mathbf{u}, \mathbf{v}) \widehat{V}\left(\mathbf{v}, \mathbf{k}_{\text {in }}\right)$ can be associated to a specific scattering pathway, as schematized in Fig. 4. In each case, the excitation is provided either by the primary incident wave $\left[\widehat{V}\left(\mathbf{v}, \mathbf{k}_{\text {in }}\right)=\hat{I}\left(\mathbf{k}_{\text {in }}, \mathbf{k}_{\text {in }}\right)\right]$ or by the secondary waves, either transmitted [ $\left.\widehat{V}\left(\mathbf{v}, \mathbf{k}_{\text {in }}\right)=\hat{T}\left(\mathbf{v}, \mathbf{k}_{\text {in }}\right)\right]$ or reflected $\left[\widehat{V}\left(\mathbf{v}, \mathbf{k}_{\text {in }}\right)=\hat{R}\left(\mathbf{v}, \mathbf{k}_{\text {in }}\right)\right]$ at the interface. On the other hand, 


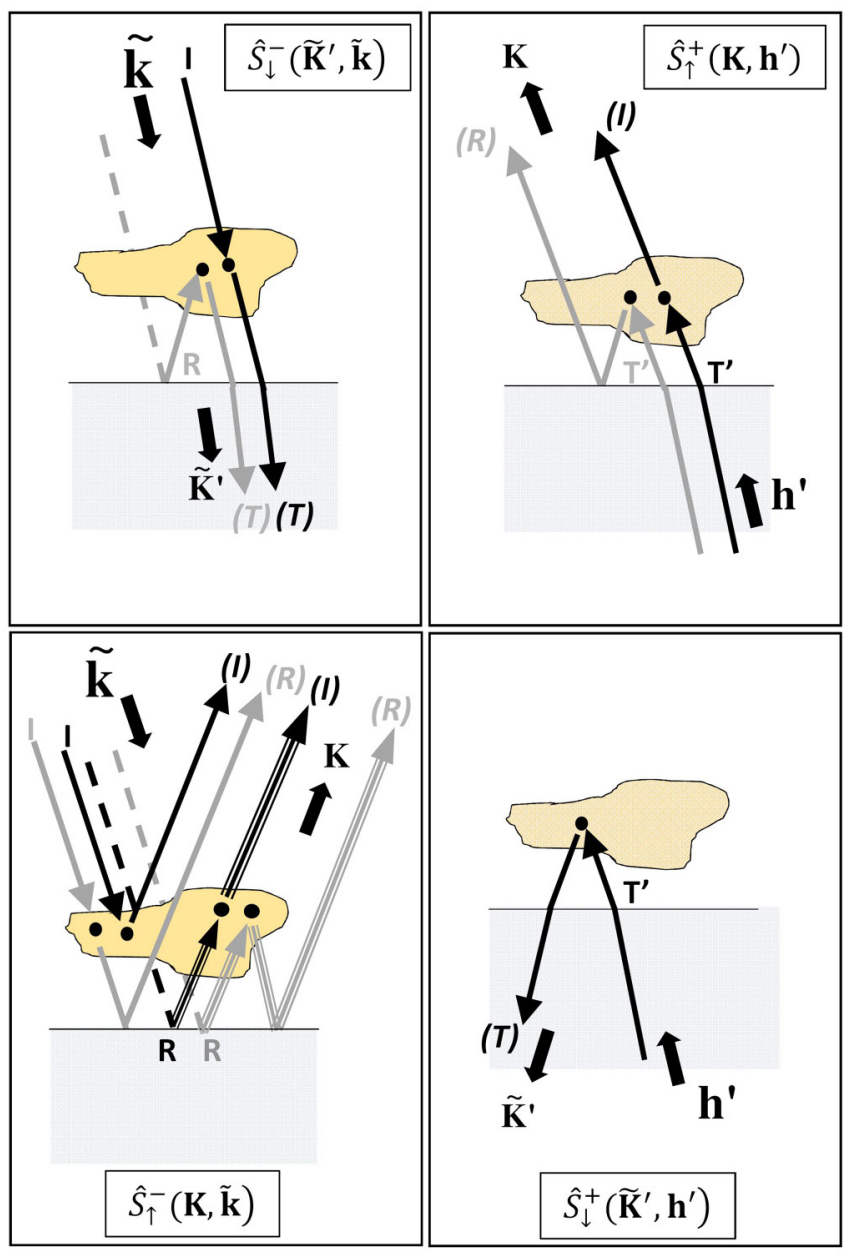

FIG. 4. Scheme of the basic mechanisms entering the four scattering operators in Eq. (19). The wave vectors of the incident and scattered waves are indicated by thick black arrows. The allowed scattering processes are indicated by different types of arrows according to the nature of the exciting and scattered waves (into brackets), with $I$ for free propagating, $R$ for reflected, and $T$ for transmitted by the interface. The black dots materialize the action of the elementary scattering operators $\hat{\Phi}(\mathbf{u}, \mathbf{v})$. The object is drawn just to materialize the scattering region above the interface.

the resulting scattered waves can propagate, either directly in the far region $\left[\widehat{W}\left(\mathbf{k}_{\mathbf{o u t}}, \mathbf{u}\right)=\hat{I}\left(\mathbf{k}_{\mathbf{o u t}}, \mathbf{k}_{\mathbf{o u t}}\right)\right]$ or can experience a prior reflection $\left[\widehat{W}\left(\mathbf{k}_{\text {out }}, \mathbf{u}\right)=\hat{R}\left(\mathbf{k}_{\text {out }}, \mathbf{u}\right)\right]$ or transmission $\left[\hat{W}\left(\mathbf{k}_{\text {out }}, \mathbf{u}\right)=\hat{T}\left(\mathbf{k}_{\text {out }}, \mathbf{u}\right)\right]$ at the interface. The wave vectors $\mathbf{u}$ and $\mathbf{v}$ are naturally and univocally connected to $\mathbf{k}_{\text {in }}$ and $\mathbf{k}_{\text {out }}$ through the constitutive definitions of the reflection and transmission operators [Eqs. (8)]. The elementary operator $\hat{\Phi}(\mathbf{u}, \mathbf{v})$ gives the vector amplitude of the far field scattered in the direction $\mathbf{u}$ as a function of the vector amplitude of a plane wave incident in the direction $\mathbf{v}$. It accounts for all the secondary excitation and scattering processes involved inside the scatterer volume. In Fig. 4, one can notice that the processes describing the forward scattering of incident waves coming from the upper and the lower half space are more symmetric than those related to backward scattering.

\section{The generalized optical theorem}

From the Maxwell's equations and considering the nature of the problem presented in Sec. IIA, the magnetic fields associated with the electric fields $\mathbf{E}^{(\text {inc/scat })}(\mathbf{r})$ are $\mathbf{H}^{(\text {inc/scat })}(\mathbf{r})=$ $-\frac{i}{\omega \mu_{0}} \nabla \times \mathbf{E}^{(i \mathrm{nc} / \mathrm{scat})}(\mathbf{r})$, and $\mathbf{H}^{\text {(tot) }}(\mathbf{r})=\mathbf{H}^{\text {(inc) }}(\mathbf{r})+\mathbf{H}^{\text {(scat) }}(\mathbf{r})$ (the reference to wave vectors $\tilde{\mathbf{k}}$ or $\mathbf{h}^{\prime}$ are omitted for simplicity). From these fields, we define the Poynting vectors

$$
\begin{aligned}
\mathbf{S}^{(\text {inc })}(\mathbf{r})= & \frac{1}{2} \operatorname{Re}\left\{\mathbf{E}^{(\text {inc })}(\mathbf{r}) \times \mathbf{H}^{(\text {inc }) *}(\mathbf{r})\right\} \\
\mathbf{S}^{(\text {scat })}(\mathbf{r})= & \frac{1}{2} \operatorname{Re}\left\{\mathbf{E}^{(\text {scat })}(\mathbf{r}) \times \mathbf{H}^{(\text {scat }) *}(\mathbf{r})\right\}, \\
\mathbf{S}^{(\mathrm{ext})}(\mathbf{r})= & \frac{1}{2} \operatorname{Re}\left\{\mathbf{E}^{(\text {inc })}(\mathbf{r}) \times \mathbf{H}^{(\text {scat }) *}(\mathbf{r})+\mathbf{E}^{(\text {scat })}(\mathbf{r})\right. \\
& \left.\times \mathbf{H}^{(\text {inc }) *}(\mathbf{r})\right\}, \\
\mathbf{S}^{(\text {tot })}(\mathbf{r})= & \frac{1}{2} \operatorname{Re}\left\{\mathbf{E}^{(\text {tot })}(\mathbf{r}) \times \mathbf{H}^{(\text {tot }) *}(\mathbf{r})\right\} \\
= & \mathbf{S}^{\text {(inc) }}(\mathbf{r})+\mathbf{S}^{(\text {scat })}(\mathbf{r})+\mathbf{S}^{(\text {ext })}(\mathbf{r}) .
\end{aligned}
$$

The absorbed, scattered and extinguished powers are given by their flux through a closed surface $\Sigma$ containing the scatterer (Fig. 2):

$$
\begin{aligned}
P^{(\mathrm{abs})} & =-\iint_{\Sigma} \mathbf{S}^{(\mathrm{tot})}(\mathbf{r}) \cdot \hat{\mathbf{n}} d^{2} S, \\
P^{(\mathrm{scat})} & =\iint_{\Sigma} \mathbf{S}^{(\mathrm{scat})}(\mathbf{r}) \cdot \hat{\mathbf{n}} d^{2} S, \\
P^{(\mathrm{ext})} & =P^{(\mathrm{abs})}+P^{(\mathrm{scat})} \\
& =-\iint_{\Sigma} \mathbf{S}^{(\mathrm{ext})}(\mathbf{r}) \cdot \hat{\boldsymbol{n}} d^{2} S .
\end{aligned}
$$

These quantities are independent of the shape and the size of $\Sigma$.

From the expressions of the scattering amplitudes [Eqs. (14)] and of the incident fields in Eqs. (3) and (4), it may be shown that, for the plane-wave excitation coming from the upper half space for instance, the total extinguished power can be written as the sum of dot products:

$$
\begin{aligned}
P^{(\mathrm{ext})-}= & \frac{2 \pi \omega \varepsilon_{0}}{k_{0}^{2}} \operatorname{Im}\left\{\left[\hat{R}(\mathbf{k}, \tilde{\mathbf{k}}) \mathbf{e}^{-}(\tilde{\mathbf{k}})\right]^{*} \cdot \mathbf{A}_{\uparrow}^{-}(\mathbf{k}, \tilde{\mathbf{k}})\right. \\
& \left.+\left[\hat{T}\left(\tilde{\mathbf{k}^{\prime}}, \tilde{\mathbf{k}}\right) \mathbf{e}^{-}(\tilde{\mathbf{k}})\right]^{*} \cdot \mathbf{A}_{\downarrow}^{-}\left(\tilde{\mathbf{k}}^{\prime}, \tilde{\mathbf{k}}\right)\right\} .
\end{aligned}
$$

By means of the stationary phase method, this result is obtained after long but straightforward algebraic manipulations based on the direct integration of the Poynting vector $\mathbf{S}^{(\mathrm{ext})}(\mathbf{r})$ over a sphere $\Sigma$ of sufficiently large radius $\left(k_{0} R \gg\right.$ 1 in Fig. 2) for using the asymptotic expressions of the far fields (see Chap. 3 in Ref [1]). It is in line with the notations of Ref. [3] which also provides a demonstration of this formula in a different approach. The vector amplitudes $\mathbf{A}_{\downarrow}^{-}\left(\tilde{\mathbf{k}}^{\prime}, \tilde{\mathbf{k}}\right)$ and $\mathbf{A}_{\uparrow}^{-}(\mathbf{k}, \tilde{\mathbf{k}})$ respectively correspond to the far field scattered in the direction of the transmitted wave $\left(\tilde{\mathbf{k}}^{\prime}\right)$ in the lower half space, and in the direction of the reflected wave (k) in the upper half space. The familiar form of the optical theorem for a plane-wave excitation $\left[P^{(\text {ext })-}=\frac{2 \pi \omega \varepsilon_{0}}{k_{0}^{2}} \operatorname{Im}\left\{\mathbf{e}^{-}(\tilde{\mathbf{k}})^{*} \cdot \mathbf{A}^{-}(\tilde{\mathbf{k}}, \tilde{\mathbf{k}})\right\}\right]$ can be recovered from Eq. (20) in the absence of a planar interface (homogeneous medium). In Eq. (20), the total extinguished power appears as the sum of the powers lost (or gained) by both the 
transmitted and reflected waves through the interface

$$
\begin{aligned}
& P_{\downarrow}^{(\mathrm{ext})-}=\frac{2 \pi \omega \varepsilon_{0}}{k_{0}^{2}} \operatorname{Im}\left\{\left[\hat{T}\left(\tilde{\mathbf{k}^{\prime}}, \tilde{\mathbf{k}}\right) \mathbf{e}^{-}(\tilde{\mathbf{k}})\right]^{*} \cdot \mathbf{A}_{\downarrow}^{-}\left(\tilde{\mathbf{k}}^{\prime}, \tilde{\mathbf{k}}\right)\right\}, \\
& P_{\uparrow}^{(\mathrm{ext})-}=\frac{2 \pi \omega \varepsilon_{0}}{k_{0}^{2}} \operatorname{Im}\left\{\left[\hat{R}(\mathbf{k}, \tilde{\mathbf{k}}) \mathbf{e}^{-}(\tilde{\mathbf{k}})\right]^{*} \cdot \mathbf{A}_{\uparrow}^{-}(\mathbf{k}, \tilde{\mathbf{k}})\right\} .
\end{aligned}
$$

The algebraic values of these quantities are controlled by the relative phase between the incident fields propagating in both half spaces (reflected field for the upper one and transmitted field for the lower one) and the far fields scattered in the same propagation directions. The generalized form of the optical theorem also holds in the case of the planewave excitation originating from the lower half space, where $P^{(\mathrm{ext})+}=P_{\uparrow}^{(\mathrm{ext})+}+P_{\downarrow}^{(\mathrm{ext})+}$, with

$$
P_{\uparrow}^{(\mathrm{ext})+}=\frac{2 \pi \omega \varepsilon_{0}}{k_{0}^{2}} \operatorname{Im}\left\{\left[\widehat{T}^{\prime}\left(\mathbf{h}, \mathbf{h}^{\prime}\right) \mathbf{e}^{+}\left(\mathbf{h}^{\prime}\right)\right]^{*} \cdot \mathbf{A}_{\uparrow}^{+}\left(\mathbf{h}, \mathbf{h}^{\prime}\right)\right\},
$$

and

$$
P_{\downarrow}^{(\mathrm{ext})+}=\frac{2 \pi \omega \varepsilon_{0}}{k_{0}^{2}} \operatorname{Im}\left\{\left[\widehat{R}^{\prime}\left(\tilde{\mathbf{h}}^{\prime}, \mathbf{h}^{\prime}\right) \mathbf{e}^{+}\left(\mathbf{h}^{\prime}\right)\right]^{*} \cdot \mathbf{A}_{\downarrow}^{+}\left(\tilde{\mathbf{h}}^{\prime}, \mathbf{h}^{\prime}\right)\right\} .
$$

Since the extinguished powers are dependent on the wave intensities, it is more convenient to consider their related cross-sections defined here as the ratio between extinguished powers and the incident powers per unit surface normal to the interface, $\hat{I}^{-}=\frac{1}{2} c \varepsilon_{0}\left|\mathbf{e}^{-}(\tilde{\mathbf{k}})\right|^{2} \frac{k_{z 1}}{k_{0}}$ or $\hat{I}^{+}=\frac{1}{2} n c \varepsilon_{0}\left|\mathbf{e}^{+}\left(\mathbf{h}^{\prime}\right)\right|^{2} \frac{h_{z 2}}{n k_{0}}$.

The relevant quantities are

$$
\begin{aligned}
C_{\downarrow}^{(\mathrm{ext})-}= & \frac{P_{\downarrow}^{(\mathrm{ext})-}}{\hat{I}^{-}}=\frac{4 \pi}{\left|\mathbf{e}^{-}(\tilde{\mathbf{k}})\right|^{2} k_{z 1}} \\
& \times \operatorname{Im}\left\{\left[\hat{T}\left(\tilde{\mathbf{k}}^{\prime}, \tilde{\mathbf{k}}\right) \mathbf{e}^{-}(\tilde{\mathbf{k}})\right]^{*} \cdot \mathbf{A}_{\downarrow}^{-}\left(\tilde{\mathbf{k}}^{\prime}, \tilde{\mathbf{k}}\right)\right\}, \\
C_{\uparrow}^{(\mathrm{ext})-}= & \frac{P_{\uparrow}^{(\mathrm{ext})-}}{\hat{I}^{-}}=\frac{4 \pi}{\left|\mathbf{e}^{-}(\tilde{\mathbf{k}})\right|^{2} k_{z 1}} \\
& \times \operatorname{Im}\left\{\left[\hat{R}(\mathbf{k}, \tilde{\mathbf{k}}) \mathbf{e}^{-}(\tilde{\mathbf{k}})\right]^{*} \cdot \mathbf{A}_{\uparrow}^{-}(\mathbf{k}, \tilde{\mathbf{k}})\right\}, \\
C_{\uparrow}^{(\mathrm{ext})+}= & \frac{P_{\uparrow}^{(\mathrm{ext})+}}{\hat{I}^{+}}=\frac{4 \pi}{\left|\mathbf{e}^{+}\left(\mathbf{h}^{\prime}\right)\right|^{2} h_{z 2}} \\
& \times \operatorname{Im}\left\{\left[\widehat{T}^{\prime}\left(\mathbf{h}, \mathbf{h}^{\prime}\right) \mathbf{e}^{+}\left(\mathbf{h}^{\prime}\right)\right]^{*} \cdot \mathbf{A}_{\uparrow}^{+}\left(\mathbf{h}, \mathbf{h}^{\prime}\right)\right\}, \\
C_{\downarrow}^{(\mathrm{ext})+}= & \frac{P_{\downarrow}^{(\mathrm{ext})+}}{\hat{I}^{+}}=\frac{4 \pi}{\left|\mathbf{e}^{+}\left(\mathbf{h}^{\prime}\right)\right|^{2} h_{z 2}} \\
& \times \operatorname{Im}\left\{\left[\widehat{R}^{\prime}\left(\tilde{\mathbf{h}}^{\prime}, \mathbf{h}^{\prime}\right) \mathbf{e}^{+}\left(\mathbf{h}^{\prime}\right)\right]^{*} \cdot \mathbf{A}_{\downarrow}^{+}\left(\tilde{\mathbf{h}}^{\prime}, \mathbf{h}^{\prime}\right)\right\},
\end{aligned}
$$

where $C^{(\mathrm{ext})-}=C_{\downarrow}^{(\mathrm{ext})-}+C_{\uparrow}^{(\mathrm{ext})-}$ and $C^{(\mathrm{ext})+}=C_{\downarrow}^{(\mathrm{ext})+}+$ $C_{\uparrow}^{\text {(ext)+ }}$ are the total extinction cross sections of the waves coming from the upper and the lower half space, respectively.

\section{EXTINCTION CROSS SECTIONS OF TRANSMITTED AND REFLECTED WAVES UNDER EXCITATIONS FROM THE UPPER OR LOWER MEDIUM}

We aim at establishing a simple connection between the extinction cross-sections of the transmitted (or reflected) waves by a supported scatterer, depending on whether it is irradiated

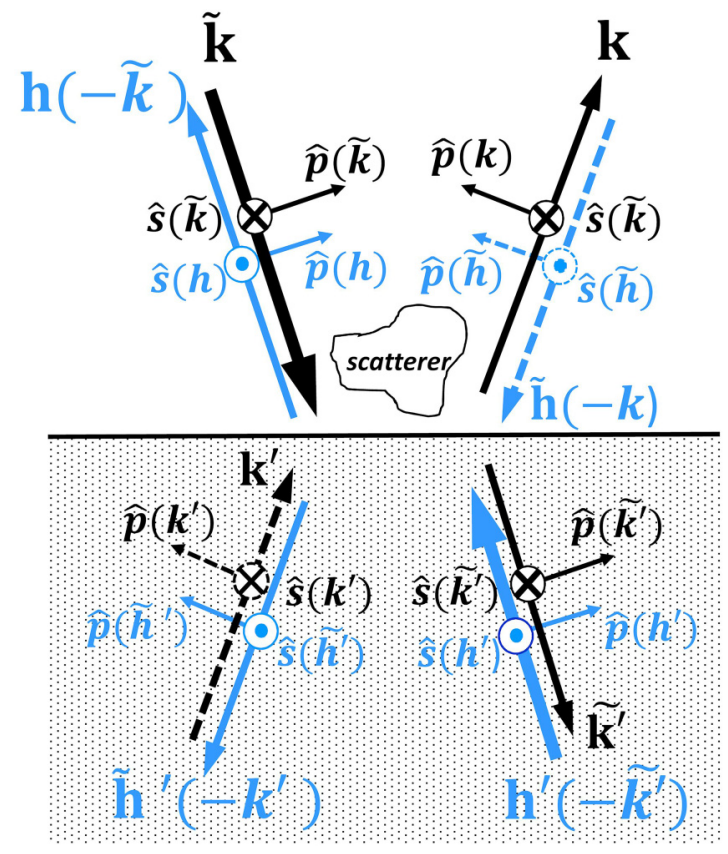

FIG. 5. Representation of incident, reflected, and transmitted wave vectors for incident plane waves generated by far sources, either in the upper half space (black) or in the lower half space (blue), chosen to develop the expressions of $C_{\downarrow}^{(\text {ext })-}$ and $C_{\uparrow}^{(\text {ext })+}$ from the generalized optical theorem formula.

from the upper half space or from the lower half space. A relevant comparison between $C_{\downarrow}^{(\mathrm{ext})-}$ and $C_{\uparrow}^{(\mathrm{ext})+}$ (or $C_{\uparrow}^{(\mathrm{ext})-}$ and $C_{\downarrow}^{(\text {ext })+}$ ) expressed for independent plane-wave excitations in Eqs. (22a) and (22c) [or Eqs. (22b) and (22d)] requires assuming, for both incident waves, a common pulsation $\omega$ and a relationship as simple as possible between their wave vectors. In line with the reciprocity of Snell's laws, the most natural choice is to set $\mathbf{h}^{\prime}=-\tilde{\mathbf{k}}^{\prime}$, and so $\mathbf{h}=-\tilde{\mathbf{k}}$. The direction of the incident wave originating from the lower medium coincides with the direction of the transmitted wave generated from the upper medium, which also implies that $\tilde{\mathbf{h}}^{\prime}=-\mathbf{k}^{\prime}$, and $\tilde{\mathbf{h}}=-\mathbf{k}$ (Fig. 5). The related pairs of incident and transmitted waves $\left(\tilde{\mathbf{k}}, \tilde{\mathbf{k}}^{\prime}\right)$ and $\left(\mathbf{h}^{\prime}=-\tilde{\mathbf{k}}^{\prime}, \mathbf{h}=-\tilde{\mathbf{k}}\right)$ define excitation schemes that will be said to be reversed one from each other in the following. Because the substrate is lossless, the basic components $k_{x}, k_{y}, k_{z 1}$, and $k_{z 2}$ involved in the various wave vectors all have real values. The quantities related to the wave coming from the lower half-space, $C_{\uparrow}^{\text {(ext)+ }}$ and $C_{\downarrow}^{(\text {ext })+}$ in Eqs. (22c) and (22d) can be written as

$$
\begin{aligned}
C_{\uparrow}^{(\mathrm{ext})+}= & \frac{4 \pi}{\left|\mathbf{e}^{+}\left(-\widetilde{\mathbf{k}^{\prime}}\right)\right|^{2} k_{z 2}} \operatorname{Im}\left\{\left[\hat{T}^{\prime}\left(-\tilde{\mathbf{k}},-\tilde{\mathbf{k}}^{\prime}\right) \mathbf{e}^{+}\left(-\tilde{\mathbf{k}}^{\prime}\right)\right]^{*}\right. \\
& \left.\cdot \mathbf{A}_{\uparrow}^{+}\left(-\tilde{\mathbf{k}},-\tilde{\mathbf{k}}^{\prime}\right)\right\},
\end{aligned}
$$

and

$$
\begin{aligned}
C_{\downarrow}^{(\mathrm{ext})+}= & \frac{4 \pi}{\left|\mathbf{e}^{+}\left(-\tilde{\mathbf{k}}^{\prime}\right)\right|^{2} k_{z 2}} \operatorname{Im}\left\{\left[\hat{R}^{\prime}\left(-\mathbf{k}^{\prime},-\tilde{\mathbf{k}}^{\prime}\right) e^{+}\left(-\tilde{\mathbf{k}}^{\prime}\right)\right]^{*}\right. \\
& \left.\cdot \mathbf{A}_{\downarrow}^{+}\left(-\mathbf{k}^{\prime},-\tilde{\mathbf{k}}^{\prime}\right)\right\} .
\end{aligned}
$$


We adopt two approaches: the first consists in developing analytically and comparing the expressions of the various cross sections in the framework of the Green's function formalism, and the second one is directly based on the application of the general principle of reciprocity in optics. If these approaches are close to each other with regard to the extinction of the transmitted waves, only the first one is really suited to discuss the extinction of reflected waves. It is important to note that the conclusions drawn below in the case of a single dielectric interface could be straightforwardly applied to the case of a scatterer close to a planar stratified medium of finite thickness. Because of the far-field formulation used here, it is sufficient to replace the transmission and reflection coefficients for a single interface with those of a layered slab and to consider that incident and transmitted waves both propagate in air ( $n=1$ for the lower half space), and in the same direction $\left(k_{z 1}=k_{z 2}, \tilde{\mathbf{k}}=\tilde{\mathbf{k}}^{\prime}\right.$, and $\left.\mathbf{k}=\mathbf{k}^{\prime}\right)$.

\section{A. The Green's function formalism}

Discussing the conditions for a possible invariance of cross sections $C_{\uparrow}^{\text {(ext)+ }}$ and $C_{\downarrow}^{(\text {ext)- }}$ in transmission (or $C_{\downarrow}^{\text {(ext) }+}$ and $C_{\uparrow}^{\text {(ext) }-}$ in reflection) first requires us to express the scattering amplitude vectors in Eqs. (14) as a function of the problem parameters (scatterer dielectric susceptibility, incident wave structures, substrate refractive index, ...). Assuming reversed excitation schemes $\left(\mathbf{h}^{\prime}=-\tilde{\mathbf{k}}^{\prime}\right)$, the four relevant scattering vector amplitudes in Eqs. (15) are written

$$
\begin{aligned}
\mathbf{A}_{\downarrow}^{-}\left(\tilde{\mathbf{k}}^{\prime}, \tilde{\mathbf{k}}\right) & =\hat{S}_{\downarrow}^{-}\left(\tilde{\mathbf{k}}^{\prime}, \tilde{\mathbf{k}}\right) \mathbf{e}^{-}(\tilde{\mathbf{k}}), \\
\mathbf{A}_{\uparrow}^{-}(\mathbf{k}, \tilde{\mathbf{k}}) & =\hat{S}_{\uparrow}^{-}(\mathbf{k}, \tilde{\mathbf{k}}) \mathbf{e}^{-}(\tilde{\mathbf{k}}), \\
\mathbf{A}_{\downarrow}^{+}\left(-\mathbf{k}^{\prime},-\tilde{\mathbf{k}}^{\prime}\right) & =\hat{S}_{\downarrow}^{+}\left(-\mathbf{k}^{\prime},-\tilde{\mathbf{k}}^{\prime}\right) \mathbf{e}^{+}\left(-\tilde{\mathbf{k}}^{\prime}\right), \\
\mathbf{A}_{\uparrow}^{+}\left(-\tilde{\mathbf{k}},-\tilde{\mathbf{k}}^{\prime}\right) & =\hat{S}_{\uparrow}^{+}\left(-\tilde{\mathbf{k}},-\tilde{\mathbf{k}}^{\prime}\right) \mathbf{e}^{+}\left(-\tilde{\mathbf{k}}^{\prime}\right),
\end{aligned}
$$

and the four corresponding scattering operators [Eqs. (19)] can be expressed as

$$
\begin{aligned}
\hat{S}_{\downarrow}^{-}\left(\tilde{\mathbf{k}}^{\prime}, \tilde{\mathbf{k}}\right)= & \frac{k_{z 2}}{k_{z 1}}\left[\hat{T}\left(\tilde{\mathbf{k}}^{\prime}, \tilde{\mathbf{k}}\right) \hat{\Phi}(\tilde{\mathbf{k}}, \tilde{\mathbf{k}}) \hat{I}(\tilde{\mathbf{k}}, \tilde{\mathbf{k}})\right. \\
& \left.+\hat{T}\left(\tilde{\mathbf{k}}^{\prime}, \tilde{\mathbf{k}}\right) \hat{\Phi}(\tilde{\mathbf{k}}, \mathbf{k}) \hat{R}(\mathbf{k}, \tilde{\mathbf{k}})\right], \\
\hat{S}_{\uparrow}^{+}\left(-\tilde{\mathbf{k}},-\tilde{\mathbf{k}}^{\prime}\right)= & \frac{k_{z 2}\left(\hat{T}\left(\tilde{\mathbf{k}}^{\prime}, \tilde{\mathbf{k}}\right) \hat{\Phi}(\tilde{\mathbf{k}}, \mathbf{k}) \hat{R}(\mathbf{k}, \tilde{\mathbf{k}})\right.}{k_{z 1}}\left(\tilde{T}\left(\tilde{\mathbf{k}}^{\prime}, \tilde{\mathbf{k}}\right) \hat{\Phi}(\tilde{\mathbf{k}}, \tilde{\mathbf{k}}) \hat{I}(\tilde{\mathbf{k}}, \tilde{\mathbf{k}})\right]^{\mathrm{T}}
\end{aligned}
$$

for forward scattering and

$$
\begin{aligned}
\hat{S}_{\uparrow}^{-}(\mathbf{k}, \tilde{\mathbf{k}})= & {[\hat{R}(\mathbf{k}, \tilde{\mathbf{k}}) \hat{\Phi}(\tilde{\mathbf{k}}, \tilde{\mathbf{k}}) \hat{I}(\tilde{\mathbf{k}}, \tilde{\mathbf{k}})} \\
& +\hat{R}(\mathbf{k}, \tilde{\mathbf{k}}) \hat{\Phi}(\tilde{\mathbf{k}}, \mathbf{k}) \hat{R}(\mathbf{k}, \tilde{\mathbf{k}}) \\
& +\hat{I}(\mathbf{k}, \mathbf{k}) \hat{\Phi}(\mathbf{k}, \tilde{\mathbf{k}}) \tilde{I}(\tilde{\mathbf{k}}, \tilde{\mathbf{k}}) \\
& +\hat{I}(\mathbf{k}, \mathbf{k}) \hat{\Phi}(\mathbf{k}, \mathbf{k}) \hat{R}(\mathbf{k}, \tilde{\mathbf{k}})], \\
\hat{S}_{\downarrow}^{+}\left(-\mathbf{k}^{\prime},-\tilde{\mathbf{k}}^{\prime}\right)= & \left(\frac{k_{z 2}}{k_{z 1}}\right)^{2}\left[\hat{T}\left(\tilde{\mathbf{k}}^{\prime}, \tilde{\mathbf{k}}\right) \hat{\Phi}(\tilde{\mathbf{k}}, \mathbf{k}) \hat{T}\left(\mathbf{k}, \mathbf{k}^{\prime}\right)\right]^{T}
\end{aligned}
$$

for backward scattering (the superscript $T$ indicates the transposition operation noticing that $[\hat{U} \hat{V}]^{\mathrm{T}}=\hat{V}^{\mathrm{T}} \hat{U}^{\mathrm{T}}$ ).

The above formulations make use of several simplifications based on reciprocity relations. The first one is
$\hat{\Phi}\left(\mathbf{k}_{2}, \mathbf{k}_{1}\right)^{\mathrm{T}}=\hat{\Phi}\left(-\mathbf{k}_{1},-\mathbf{k}_{2}\right)$, which is a direct consequence of the basic reciprocity property of the Green's operators $\left[\hat{G}\left(\mathbf{r}, \mathbf{r}^{\prime}\right)=\hat{G}\left(\mathbf{r}^{\prime}, \mathbf{r}\right)^{T}\right.$ and $\left.\hat{\mathcal{G}}^{i}\left(\mathbf{r}, \mathbf{r}_{i}\right)=\hat{\mathcal{G}}^{i}\left(\mathbf{r}_{i}, \mathbf{r}\right)^{T}\right]$, as shown in Appendix D. This property remains valid in the exact formulation of the effective Green's operator accounting for singularities in the scatterer volume. The second ones directly result from the definition of the transmission and reflection dyadics in Eqs. (8), which allows us to write $\hat{T}\left(\mathbf{k}_{2}, \mathbf{k}_{1}\right)=$ $\hat{T}\left(-\mathbf{k}_{2},-\mathbf{k}_{1}\right)=\hat{T}\left(\mathbf{k}_{1}, \mathbf{k}_{2}\right)^{T}$ and $\hat{R}\left(\mathbf{k}_{2}, \mathbf{k}_{1}\right)=\hat{R}\left(-\mathbf{k}_{2},-\mathbf{k}_{1}\right)=$ $\hat{R}\left(\mathbf{k}_{1}, \mathbf{k}_{2}\right)^{T}$ for any pair $\left(\mathbf{k}_{2}, \mathbf{k}_{1}\right)$ of connected wave vectors. The last one relates to the transmission operators of ascending and descending incident waves through the relation

$$
\hat{T}^{\prime}\left(-\tilde{\mathbf{k}},-\tilde{\mathbf{k}}^{\prime}\right)=\frac{k_{z 2}}{k_{z 1}}\left[\hat{T}\left(\tilde{\mathbf{k}}^{\prime}, \tilde{\mathbf{k}}\right)\right]^{\mathrm{T}}=\frac{k_{z 2}}{k_{z 1}} \hat{T}\left(\tilde{\mathbf{k}}, \tilde{\mathbf{k}}^{\prime}\right),
$$

making use of the relation $t_{s / p}^{\prime}\left(k_{\|}\right)=\frac{k_{z 2}}{k_{z 1}} t_{s / p}\left(k_{\|}\right)$given by the Snell-Descartes laws (Appendix A). A comparison between Eqs. (25a) and (25b) allows us to establish the important reciprocity relation between the forward-scattering operators:

$$
\hat{S}_{\uparrow}^{+}\left(-\tilde{\mathbf{k}},-\tilde{\mathbf{k}}^{\prime}\right)=\hat{S}_{\downarrow}^{-}\left(\tilde{\mathbf{k}}^{\prime}, \tilde{\mathbf{k}}\right)^{T},
$$

whereas no similar relation between the backward-scattering operators can be obtained.

\section{Extinction of the transmitted waves: Conditions for an invariance relative to the direction of illumination}

We want to discuss here under which conditions the extinction cross sections for the transmitted waves $\left(C_{\downarrow}^{\text {(ext)- }}\right.$ and $C_{\uparrow}^{(\text {ext })+}$ ) may be identical. Assuming that the incidence and the polarization state $\left[\mathbf{e}^{-}(\tilde{\mathbf{k}})=e_{s}^{-}(\tilde{\mathbf{k}}) \hat{\boldsymbol{s}}(\tilde{\mathbf{k}})+e_{p}^{-}(\tilde{\mathbf{k}}) \hat{\boldsymbol{p}}(\tilde{\mathbf{k}})\right]$ of the excitation wave coming from the upper half-space [Eq. (4a)] are fixed, the incidence of the excitation wave coming from the lower half-space is determined by the above-mentioned condition $\mathbf{h}^{\prime}=-\tilde{\mathbf{k}}^{\prime}$ and its polarization state $\left[\mathbf{e}^{+}\left(-\tilde{\mathbf{k}}^{\prime}\right)=e_{s}^{+}\left(-\tilde{\mathbf{k}}^{\prime}\right) \hat{\boldsymbol{s}}\left(-\tilde{\mathbf{k}}^{\prime}\right)+e_{p}^{+}\left(-\tilde{\mathbf{k}}^{\prime}\right) \hat{\boldsymbol{p}}\left(-\tilde{\mathbf{k}}^{\prime}\right)\right]$ is the only adjustable parameter to consider for achieving $C_{\downarrow}^{(\text {ext })-}=$ $C_{\uparrow}^{\text {(ext)+ }}$ that can be alternatively expressed, from Eqs. (22a) and (23a), in the form

$$
\begin{aligned}
\frac{4 \pi}{\left|\mathbf{e}^{-}(\tilde{\mathbf{k}})\right|^{2} k_{z 1}} \operatorname{Im}\left\{\left[\hat{T}\left(\tilde{\mathbf{k}}^{\prime}, \tilde{\mathbf{k}}\right) \mathbf{e}^{-}(\tilde{\mathbf{k}})\right]^{*} \cdot \mathbf{A}_{\downarrow}^{-}\left(\tilde{\mathbf{k}}^{\prime}, \tilde{\mathbf{k}}\right)\right\} \\
=\frac{4 \pi}{\left|\mathbf{e}^{+}\left(-\tilde{\mathbf{k}}^{\prime}\right)\right|^{2} k_{z 2}} \operatorname{Im}\left\{\left[\hat{T}^{\prime}\left(-\tilde{\mathbf{k}},-\tilde{\mathbf{k}}^{\prime}\right) \mathbf{e}^{+}\left(-\tilde{\mathbf{k}}^{\prime}\right)\right]^{*}\right. \\
\left.\quad \cdot \mathbf{A}_{\uparrow}^{+}\left(-\tilde{\mathbf{k}},-\tilde{\mathbf{k}}^{\prime}\right)\right\},
\end{aligned}
$$

or, after introducing the scattering operators [Eqs. (25a) and (25b)],

$$
\begin{aligned}
& \frac{4 \pi}{\left|\mathbf{e}^{-}(\tilde{\mathbf{k}})\right|^{2} k_{z 1}} \operatorname{Im}\left\{\left[\hat{T}\left(\tilde{\mathbf{k}}^{\prime}, \tilde{\mathbf{k}}\right) \mathbf{e}^{-}(\tilde{\mathbf{k}})\right]^{*} \cdot \hat{S}_{\downarrow}^{-}\left(\tilde{\mathbf{k}}^{\prime}, \tilde{\mathbf{k}}\right) \mathbf{e}^{-}(\tilde{\mathbf{k}})\right\} \\
& =\frac{4 \pi}{\left|\mathbf{e}^{+}\left(-\tilde{\mathbf{k}}^{\prime}\right)\right|^{2} k_{z 2}} \operatorname{Im}\left\{\left[\hat{T}^{\prime}\left(-\tilde{\mathbf{k}},-\tilde{\mathbf{k}}^{\prime}\right) \mathbf{e}^{+}\left(-\tilde{\mathbf{k}}^{\prime}\right)\right]^{*}\right. \\
& \left.\quad \cdot S_{\uparrow}^{+}\left(-\tilde{\mathbf{k}},-\tilde{\mathbf{k}}^{\prime}\right) \mathbf{e}^{+}\left(-\tilde{\mathbf{k}}^{\prime}\right)\right\} .
\end{aligned}
$$

If a polarization state $\mathbf{e}^{+}\left(-\tilde{\mathbf{k}}^{\prime}\right)$ is a solution of Eq. (28b), it is reasonable to believe, without further proof, that it should 
also be a solution of the same equation involving the real parts of the quantities into brackets since it must be independent of the excitation wavelength and of the complex electric susceptibility $\eta(\mathbf{r})$ of the scatterer. Equation (28b) can be generalized in the form

$$
\begin{aligned}
\frac{4 \pi}{\left|\mathbf{e}^{-}(\tilde{\mathbf{k}})\right|^{2} k_{z 1}}\left[\hat{T}\left(\tilde{\mathbf{k}}^{\prime}, \tilde{\mathbf{k}}\right) \mathbf{e}^{-}(\tilde{\mathbf{k}})\right]^{*} \cdot \hat{S}_{\downarrow}^{-}\left(\tilde{\mathbf{k}}^{\prime}, \tilde{\mathbf{k}}\right) \mathbf{e}^{-}(\tilde{\mathbf{k}}) \\
=\frac{4 \pi}{\left|\mathbf{e}^{+}\left(-\tilde{\mathbf{k}}^{\prime}\right)\right|^{2} k_{z 2}}\left[\hat{T}^{\prime}\left(-\tilde{\mathbf{k}},-\tilde{\mathbf{k}}^{\prime}\right) \mathbf{e}^{+}\left(-\tilde{\mathbf{k}}^{\prime}\right)\right]^{*} \\
\quad \cdot S_{\uparrow}^{+}\left(-\tilde{\mathbf{k}},-\tilde{\mathbf{k}}^{\prime}\right) \mathbf{e}^{+}\left(-\tilde{\mathbf{k}}^{\prime}\right) .
\end{aligned}
$$

A condition on $\mathbf{e}^{+}\left(-\tilde{\mathbf{k}}^{\prime}\right)$ that fulfills Eq. (28c) will be sufficient to fulfill (28b) and we assume it is also necessary. Making use of the general property $\mathbf{A} \cdot(\hat{V} \mathbf{B})=\left(\hat{V}^{\mathrm{T}} \mathbf{A}\right) \cdot \mathbf{B}$ and the reciprocity relations (26) and (27), Eq. (28c) is written

$$
\begin{aligned}
& {\left[\hat{T}\left(\tilde{\mathbf{k}}^{\prime}, \tilde{\mathbf{k}}\right) \frac{\mathbf{e}^{-}(\tilde{\mathbf{k}})}{\left|\mathbf{e}^{-}(\tilde{\mathbf{k}})\right|}\right]^{*} \cdot \hat{S}_{\downarrow}^{-}\left(\tilde{\mathbf{k}}^{\prime}, \tilde{\mathbf{k}}\right) \frac{\mathbf{e}^{-}(\tilde{\mathbf{k}})}{\left|\mathbf{e}^{-}(\tilde{\mathbf{k}})\right|}} \\
& \quad=\frac{\mathbf{e}^{+}\left(-\tilde{\mathbf{k}}^{\prime}\right)}{\left|\mathbf{e}^{+}\left(-\tilde{\mathbf{k}}^{\prime}\right)\right|} \cdot \hat{S}_{\downarrow}^{-}\left(\tilde{\mathbf{k}}^{\prime}, \tilde{\mathbf{k}}\right)\left[\hat{T}\left(\tilde{\mathbf{k}}^{\prime}, \tilde{\mathbf{k}}\right)^{T} \frac{\mathbf{e}^{+}\left(-\tilde{\mathbf{k}}^{\prime}\right)}{\left|\mathbf{e}^{+}\left(-\tilde{\mathbf{k}}^{\prime}\right)\right|}\right]^{*}
\end{aligned}
$$

The conditions for establishing Eq. (28d) are very general and do not depend on the size and the nature of the scatterer. As a consequence, Eq. (28d) must hold for any scattering operator $\hat{S}_{\downarrow}^{-}\left(\tilde{\mathbf{k}}^{\prime}, \tilde{\mathbf{k}}\right)$ which is independent of the wave polarizations. It can then only be verified provided that right and left vectors in both members of this expression are proportional with

$$
\begin{gathered}
{\left[\hat{T}(\tilde{\mathbf{k}}, \tilde{\mathbf{k}}) \frac{\mathbf{e}^{-}(\tilde{\mathbf{k}})}{\left|\mathbf{e}^{-}(\tilde{\mathbf{k}})\right|}\right]^{*}=\alpha \frac{\mathbf{e}^{+}\left(-\tilde{\mathbf{k}}^{\prime}\right)}{\left|\mathbf{e}^{+}\left(-\tilde{\mathbf{k}}^{\prime}\right)\right|} \text { and }} \\
{\left[\hat{T}\left(\tilde{\mathbf{k}}^{\prime}, \tilde{\mathbf{k}}\right)^{T} \frac{\mathbf{e}^{+}\left(-\tilde{\mathbf{k}}^{\prime}\right)}{\left|\mathbf{e}^{+}\left(-\tilde{\mathbf{k}}^{\prime}\right)\right|}\right]^{*}=\alpha \frac{\mathbf{e}^{-}(\tilde{\mathbf{k}})}{\left|\mathbf{e}^{-}(\tilde{\mathbf{k}})\right|}}
\end{gathered}
$$

where $\alpha$ is an arbitrary complex number. This leads to the equality $\mathbf{e}^{-}(\tilde{\mathbf{k}})=|\alpha|^{-2} \hat{T}\left(\tilde{\mathbf{k}}^{\prime}, \tilde{\mathbf{k}}\right)^{\mathrm{T} *} \hat{T}\left(\tilde{\mathbf{k}} \tilde{\mathbf{k}}^{\prime}, \tilde{\mathbf{k}}\right) \mathbf{e}^{-}(\tilde{\mathbf{k}})$ or, equivalently, to the statement that the operator $\hat{T}\left(\tilde{\mathbf{k}}^{\prime}, \tilde{\mathbf{k}}\right)^{\mathrm{T} *} \hat{T}\left(\tilde{\mathbf{k}}^{\prime}, \tilde{\mathbf{k}}\right)$ commutes with the projector $\hat{I}(\tilde{\mathbf{k}}, \tilde{\mathbf{k}})$. From the definition of the dyadic in Eq. (8c), we have $\hat{T}\left(\tilde{\mathbf{k}}^{\prime}, \tilde{\mathbf{k}}\right)^{\mathrm{T} *} \hat{T}\left(\tilde{\mathbf{k}}^{\prime}, \tilde{\mathbf{k}}\right)=\left|t_{s}\left(k_{\|}\right)\right|^{2} \hat{\boldsymbol{s}}(\tilde{\mathbf{k}}) \otimes \hat{\boldsymbol{s}}(\tilde{\mathbf{k}})+\left|t_{p}\left(k_{\|}\right)\right|^{2} \hat{\boldsymbol{p}}(\tilde{\mathbf{k}}) \otimes$ $\hat{\boldsymbol{p}}(\tilde{\mathbf{k}})$. The condition $\left[\hat{T}\left(\tilde{\mathbf{k}}^{\prime}, \tilde{\mathbf{k}}\right)^{\mathrm{T} *} \hat{T}\left(\tilde{\mathbf{k}}^{\prime}, \tilde{\mathbf{k}}\right), \hat{I}(\tilde{\mathbf{k}}, \tilde{\mathbf{k}})\right]=0$ is then possible either if $\left|t_{s}\left(k_{\|}\right)\right|^{2}=\left|t_{p}\left(k_{\|}\right)\right|^{2}$, which corresponds to the case of a normal incidence relative to the interface $\left[k_{||}=0, t=t_{p}\left(k_{||}\right)=t_{s}\left(k_{||}\right)\right]$, or if $\left|t_{s}\left(k_{||}\right)\right|^{2}=0$ (alternatively $\left|t_{p}\left(k_{||}\right)\right|^{2}=0$ ), which corresponds to the case of a pure TE (alternatively TM) polarization of the incident wave. As a general rule, $\left[\hat{T}\left(\tilde{\mathbf{k}}^{\prime}, \tilde{\mathbf{k}}\right)^{\mathrm{T} *} \hat{T}\left(\tilde{\mathbf{k}}^{\prime}, \tilde{\mathbf{k}}\right), \hat{I}(\tilde{\mathbf{k}}, \tilde{\mathbf{k}})\right] \neq 0$ for an arbitrary polarized wave at oblique incidence $\left(t_{s}\left(k_{||}\right) \neq t_{p}\left(k_{||}\right)\right)$, implying that the extinction of the transmitted waves cannot be invariant under a permutation of the directions of illumination $\left(C_{\downarrow}^{(\mathrm{ext})-} \neq C_{\uparrow}^{(\mathrm{ext})+}\right)$. This constitutes the key result of this analysis.

In the case of normal incidence, $\alpha=\operatorname{te}^{i \beta}$ (where $\beta$ is an arbitrary real number), it turns out that $\hat{T}\left(\tilde{\mathbf{k}}^{\prime}, \tilde{\mathbf{k}}\right) \mathbf{e}^{-}(\tilde{\mathbf{k}})=t \mathbf{e}^{-}(\tilde{\mathbf{k}})$ and we obtain, from Eq. (29),

$$
\frac{\mathbf{e}^{+}\left(-\tilde{\mathbf{k}}^{\prime}\right)}{\left|\mathbf{e}^{+}\left(-\tilde{\mathbf{k}}^{\prime}\right)\right|}=\frac{\mathbf{e}^{-*}(\tilde{\mathbf{k}})}{\left|\mathbf{e}^{-}(\tilde{\mathbf{k}})\right|} e^{-i \beta},
$$

In the case of pure TE or TM waves at oblique incidence $\hat{T}\left(\tilde{\mathbf{k}}^{\prime}, \tilde{\mathbf{k}}\right)=t_{s}\left(k_{\|}\right) \hat{\boldsymbol{S}}\left(\tilde{\mathbf{k}}^{\prime}\right) \otimes \hat{\boldsymbol{s}}(\tilde{\mathbf{k}})\left(\alpha=t_{s}\left(k_{\|}\right) e^{i \beta_{\mathrm{TE}}}\right)$ or $\hat{T}\left(\tilde{\mathbf{k}}^{\prime}, \tilde{\mathbf{k}}\right)=$ $t_{p}\left(k_{\|}\right) \hat{\boldsymbol{p}}\left(\tilde{\mathbf{k}}^{\prime}\right) \otimes \hat{\boldsymbol{p}}(\tilde{\mathbf{k}})\left[\alpha=t_{p}\left(k_{\|}\right) e^{i \beta_{\mathrm{TM}}}\right]$. We then have, from Eq. (29) and according to the notations in Eq. (4), the general relation

$$
\begin{aligned}
\frac{\mathbf{e}^{+}\left(-\tilde{\mathbf{k}}^{\prime}\right)}{\left|\mathbf{e}^{+}\left(-\tilde{\mathbf{k}}^{\prime}\right)\right|} & =\alpha^{-1} \hat{T}\left(\tilde{\mathbf{k}}^{\prime}, \tilde{\mathbf{k}}\right)^{*} \frac{\mathbf{e}^{-*}(\tilde{\mathbf{k}})}{\left|\mathbf{e}^{-}(\tilde{\mathbf{k}})\right|}=\alpha^{-1} \frac{\mathbf{e}^{(\mathbf{t})-*}\left(\tilde{\mathbf{k}}^{\prime}\right)}{\left|\mathbf{e}^{-}(\tilde{\mathbf{k}})\right|} \\
& =\frac{\mathbf{e}^{(\mathbf{t})-*}\left(\tilde{\mathbf{k}}^{\prime}\right)}{\left|\mathbf{e}^{(\mathbf{t})-}\left(\tilde{\mathbf{k}}^{\prime}\right)\right|} \mathrm{e}^{-i \beta_{\mathrm{TM} / \mathrm{TE}}} .
\end{aligned}
$$

Relations (30) and (31) are equivalent in the case of normal incidence where incident and transmitted wave vectors and polarizations are proportional $\left[\tilde{\mathbf{k}}^{\prime}=n \tilde{\mathbf{k}}\right.$ and $\mathbf{e}^{(t)-}\left(\tilde{\mathbf{k}}^{\prime}\right)=$ $\left.t \mathbf{e}^{-}(\tilde{\mathbf{k}})\right]$.

In both favorable cases discussed above, the condition ensuring, for any scatterer and any wavelength, the invariance of the optical extinction cross sections in transmission for reversed-excitation schemes, amounts to stating that the polarization of the incident wave propagating from the lower half space must be the complex conjugate of the polarization of the transmitted wave coming from the upper half space, to an arbitrary phase factor which is intended to vanish in the formulation of the cross-sections. When the waves are linearly polarized, complex conjugation is not necessary since it can be rooted in the choice of this arbitrary phase.

\section{Extinction of the reflected waves: Sensitivity to the direction of illumination}

We examine here the possibility that the extinction processes related to waves reflected at the interface may be also invariant for reversed excitation schemes. The corresponding cross sections to be compared are given in Eqs. (22b) and (23b):

$$
C_{\uparrow}^{(\mathrm{ext})-}=\frac{4 \pi}{\left|\mathbf{e}^{-}(\tilde{\mathbf{k}})\right|^{2} k_{z 1}} \operatorname{Im}\left\{\left[\hat{R}(\mathbf{k}, \tilde{\mathbf{k}}) \mathbf{e}^{-}(\tilde{\mathbf{k}})\right]^{*} \cdot \hat{S}_{\uparrow}^{-}(\mathbf{k}, \tilde{\mathbf{k}}) \mathbf{e}^{-}(\tilde{\mathbf{k}})\right\},
$$

and

$$
\begin{aligned}
C_{\downarrow}^{(\mathrm{ext})+}= & \frac{4 \pi}{\left|\mathbf{e}^{+}\left(-\tilde{\mathbf{k}}^{\prime}\right)\right|^{2} k_{z 2}} \operatorname{Im}\left\{\left[\hat{R}^{\prime}\left(-\mathbf{k}^{\prime},-\tilde{\mathbf{k}}^{\prime}\right) \mathbf{e}^{+}\left(-\tilde{\mathbf{k}}^{\prime}\right)\right]^{*}\right. \\
& \left.\cdot \hat{S}_{\downarrow}^{+}\left(-\mathbf{k}^{\prime},-\tilde{\mathbf{k}}^{\prime}\right) \mathbf{e}^{+}\left(-\tilde{\mathbf{k}}^{\prime}\right)\right\} .
\end{aligned}
$$

Since such an invariance cannot be achieved for the extinction of transmitted waves in the general case of an arbitrary polarized fields at oblique incidence, the strong differences in the formulations of $\hat{S}_{\uparrow}^{-}(\mathbf{k}, \tilde{\mathbf{k}})$ and $\hat{S}_{\downarrow}^{+}\left(-\mathbf{k}^{\prime},-\tilde{\mathbf{k}}^{\prime}\right)$ [Eqs. (25c) and (25d)] even more precludes this possibility for reflected waves. Let us rather consider the simpler case of an excitation at normal incidence $\left(\tilde{\mathbf{k}}=-\mathbf{k}=-k_{z 1} \hat{\mathbf{z}}, \quad-\tilde{\mathbf{k}}^{\prime}=k_{z 2} \hat{\mathbf{z}}, \quad k_{z 1}=k_{0}, \quad\right.$ and $\left.k_{z 2}=n k_{0}\right)$. Transmission and reflection coefficients are strictly independent of the polarization of the transverse electric field 
$\left[t_{s}\left(k_{\|}=0\right)=t_{p}\left(k_{\|}=0\right)=t, r_{p}\left(k_{\|}=0\right)=-r_{s}\left(k_{\|}=0\right)=r\right]$ leading to $\hat{R}(\mathbf{k}=-\tilde{\mathbf{k}}, \tilde{\mathbf{k}})=-r \hat{I}(\tilde{\mathbf{k}}, \tilde{\mathbf{k}}), \hat{T}\left(\tilde{\mathbf{k}}^{\prime}, \tilde{\mathbf{k}}\right)=t \hat{I}(\tilde{\mathbf{k}}, \tilde{\mathbf{k}})$, and $\hat{T}\left(\mathbf{k}, \mathbf{k}^{\prime}\right)=\hat{T}\left(-\widetilde{\mathbf{k}},-\tilde{\mathbf{k}}^{\prime}\right)=\hat{T}\left(\tilde{\mathbf{k}}^{\prime}, \tilde{\mathbf{k}}\right)^{T}$. Noticing that $\hat{I}(\tilde{\mathbf{k}}, \tilde{\mathbf{k}})^{2}=\hat{I}(\tilde{\mathbf{k}}, \tilde{\mathbf{k}}), \quad \hat{I}(\tilde{\mathbf{k}}, \tilde{\mathbf{k}})^{T}=\hat{I}(\tilde{\mathbf{k}}, \tilde{\mathbf{k}})=\hat{I}(-\tilde{\mathbf{k}},-\tilde{\mathbf{k}}), \quad$ and $\hat{\Phi}(\tilde{\mathbf{k}}, \mathbf{k}=-\tilde{\mathbf{k}})^{T}=\hat{\Phi}(\mathbf{k}=-\tilde{\mathbf{k}}, \tilde{\mathbf{k}})$, Eqs. (25c) and (25d) simplify in the form

$$
\begin{aligned}
\hat{S}_{\uparrow}^{-}(\mathbf{k}=-\tilde{\mathbf{k}}, \tilde{\mathbf{k}})= & {[-r \hat{I}(\tilde{\mathbf{k}}, \tilde{\mathbf{k}}) \hat{\Phi}(\tilde{\mathbf{k}}, \tilde{\mathbf{k}}) \hat{I}(\tilde{\mathbf{k}}, \tilde{\mathbf{k}})} \\
& +r^{2} \hat{I}(\tilde{\mathbf{k}}, \tilde{\mathbf{k}}) \hat{\Phi}(\tilde{\mathbf{k}},-\tilde{\mathbf{k}}) \hat{I}(\tilde{\mathbf{k}}, \tilde{\mathbf{k}}) \\
& +\hat{I}(\tilde{\mathbf{k}}, \tilde{\mathbf{k}}) \hat{\Phi}(-\tilde{\mathbf{k}}, \tilde{\mathbf{k}}) \hat{I}(\tilde{\mathbf{k}}, \tilde{\mathbf{k}}) \\
& -r \hat{I}(\tilde{\mathbf{k}}, \tilde{\mathbf{k}}) \hat{\Phi}(-\tilde{\mathbf{k}},-\tilde{\mathbf{k}}) \hat{I}(\tilde{\mathbf{k}}, \tilde{\mathbf{k}})]
\end{aligned}
$$

and

$$
\hat{S}_{\downarrow}^{+}\left(-\mathbf{k}^{\prime}=\tilde{\mathbf{k}}^{\prime},-\tilde{\mathbf{k}}^{\prime}\right)=(n t)^{2}[\hat{I}(\tilde{\mathbf{k}}, \tilde{\mathbf{k}}) \hat{\Phi}(\tilde{\mathbf{k}},-\tilde{\mathbf{k}}) \hat{I}(\tilde{\mathbf{k}}, \tilde{\mathbf{k}})]
$$

Backward-scattering operators are basically too different to ensure, even under any further conditions, the general equality between the cross sections $C_{\uparrow}^{\text {(ext)- }}$ and $C_{\downarrow}^{\text {(ext)+ }}$. The same conclusion could be drawn for pure TE or TM excitations at oblique incidence, which leads to the general conclusion that the extinction of reflected waves and therefore the total extinction itself are always sensitive to the way the supported scatterer is illuminated, either from the upper or from the lower medium. This is particularly apparent in the simple case of a normal incidence under the hypothesis that the height of the scatterer in the direction normal to the substrate $(\Delta z)$ is small compared with the wavelength $\left(\Delta z k_{0} \ll 2 \pi\right)$. In that case, the phase factors in the expression (18) can be neglected, which yields $\hat{\Phi}(\tilde{\mathbf{k}}, \tilde{\mathbf{k}}) \cong \hat{\Phi}(\tilde{\mathbf{k}},-\tilde{\mathbf{k}}) \cong \hat{\Phi}(-\tilde{\mathbf{k}}, \tilde{\mathbf{k}}) \cong$ $\hat{\Phi}(-\tilde{\mathbf{k}},-\tilde{\mathbf{k}}) \cong \hat{\Phi}(\tilde{\mathbf{k}}, \tilde{\mathbf{k}})^{T}$ and therefore $\hat{S}_{\downarrow}^{+}\left(\mathbf{k}^{\prime}=-\tilde{\mathbf{k}},-\tilde{\mathbf{k}}^{\prime}\right) \cong$ $\left(\frac{n t}{1-r}\right)^{2} \hat{S}_{\uparrow}^{-}(\mathbf{k}=-\tilde{\mathbf{k}}, \tilde{\mathbf{k}})=n^{2} \hat{S}_{\uparrow}^{-}(\mathbf{k}=-\tilde{\mathbf{k}}, \tilde{\mathbf{k}})$, since $t=1-r$ for real reflection- and transmission-type coefficients at an interface (Appendix A). From Eqs. (8a) and (8b) and noticing that $\hat{\boldsymbol{s}}\left(\tilde{\mathbf{k}}^{\prime}\right)=\hat{\boldsymbol{s}}(\tilde{\mathbf{k}})$ and $\hat{\boldsymbol{p}}\left(\tilde{\mathbf{k}}^{\prime}\right)=\hat{\boldsymbol{p}}(\tilde{\mathbf{k}})$ for a normal incidence (Fig. 5), the reflection operators are written $\hat{R}(\mathbf{k}=-\tilde{\mathbf{k}}, \tilde{\mathbf{k}})=$ $r \hat{I}(\tilde{\mathbf{k}}, \tilde{\mathbf{k}})$ and $\hat{R}^{\prime}\left(-\mathbf{k}^{\prime}=\tilde{\mathbf{k}}^{\prime},-\tilde{\mathbf{k}}\right)=r^{\prime} \hat{I}(\tilde{\mathbf{k}}, \tilde{\mathbf{k}})$ in Eqs. (33), with $r=-r^{\prime}$ (Appendix A). No general relation between $\mathbf{e}^{+}\left(-\tilde{\mathbf{k}}^{\prime}\right)$ and $\mathbf{e}^{-}(\tilde{\mathbf{k}})$ allows us to establish the equality $C_{\uparrow}^{\text {(ext)- }}=C_{\downarrow}^{\text {(ext)+ }}$ from Eqs. (32). It nevertheless appears that, if $\frac{\mathbf{e}^{+}\left(-\tilde{\mathbf{k}}^{\prime}\right)}{\left|\mathbf{e}^{+}\left(-\tilde{\mathbf{k}}^{\prime}\right)\right|}=$ $\frac{\mathbf{e}^{-}(\tilde{\mathbf{K}})}{\left|\mathbf{e}^{-}(\tilde{\mathbf{k}})\right|} e^{i \beta}$ (arbitrary real $\beta$ value), the profiles of the extinction cross-sections in reflection are almost homothetic, with opposite signs $\left(C_{\downarrow}^{(\mathrm{ext})+} \cong-n C_{\uparrow}^{\text {(ext)- }}\right)$. It must be noted that this condition differs from Eq. (30) in the case of an elliptically polarized incident wave.

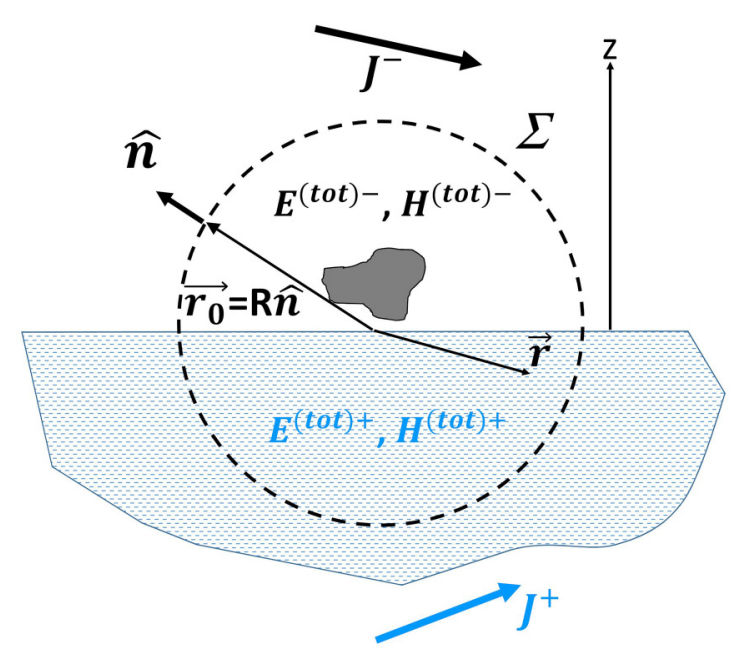

FIG. 6. System consisting of a scatterer above a plane interface separating two semi-infinite media (air and dielectric). $\boldsymbol{J}^{-}$and $\boldsymbol{J}^{+}$ are the source currents that generate plane-wave excitations and the respective total electromagnetic fields $\left[\mathbf{E}^{(\text {tot })-}(\mathbf{r}), \mathbf{H}^{(\text {tot })-}(\mathbf{r})\right]$ and $\left[\mathbf{E}^{(\mathrm{tot})+}(\mathbf{r}), \mathbf{H}^{(\mathrm{tot})+}(\mathbf{r})\right]$ for any position $\mathbf{r}$. A sphere $\Sigma$ of very large radius $R$ compared with the wavelength encloses the scatterer.

\section{B. The Lorentz reciprocity theorem}

The basic conditions that ensure the invariance of the extinction cross sections of the transmitted light for reversed excitation schemes can be defined through an alternative approach, based on the Lorentz reciprocity theorem [20]. Considering the system pictured in Fig. 2, the excitation plane waves coming from the upper and lower half spaces are assumed to be created respectively by electric current source densities $\boldsymbol{J}^{-}$and $\boldsymbol{J}^{+}$located at infinite distances from the interface, outside of a volume $V$ (outer surface $\Sigma$ ) enclosing the scatterer (Fig. 6). The total field in the presence of $\boldsymbol{J}^{-}$(respectively $\left.\boldsymbol{J}^{+}\right)$is denoted $\left[\mathbf{E}^{(\text {tot })}(\mathbf{r}), \mathbf{H}^{(\text {tot })-}(\mathbf{r})\right]$ [respectively $\left.\left[\mathbf{E}^{\text {(tot) })}(\mathbf{r}), \mathbf{H}^{(\mathrm{tot})+}(\mathbf{r})\right]\right]$.

The electric fields are split into two contributions, one corresponding to the direct excitation by the sources, including reflected and transmitted plane waves, and one corresponding to the field scattered by the particle. According to the notations in Sec. II, $\mathbf{E}^{(\text {tot })-}(\tilde{\mathbf{k}}, \mathbf{r})=\mathbf{E}^{(\mathrm{inc})-}(\tilde{\mathbf{k}}, \mathbf{r})+\mathbf{E}^{\text {(scat) })}(\tilde{\mathbf{k}}, \mathbf{r}) \quad$ and $\quad \mathbf{E}^{(\text {tot })+}$ $\left(\mathbf{h}^{\prime}, \mathbf{r}\right)=\mathbf{E}^{\text {(inc) })+}\left(\mathbf{h}^{\prime}, \mathbf{r}\right)+\mathbf{E}^{(\text {scat })+}\left(\mathbf{h}^{\prime}, \mathbf{r}\right)$, where the incident fields can be fully expressed through Eqs. (3) and (4). The magnetic fields are obtained from the electric fields through Maxwell's equations in the source-free region $\left[\mathbf{H}^{(\mathrm{tot}) \pm}(\mathbf{r})=\frac{-i}{\omega \mu_{0}} \boldsymbol{\nabla} \times \mathbf{E}^{(\mathrm{tot}) \pm}(\mathbf{r})\right]$. In this framework, since $\boldsymbol{J}^{-}(\mathbf{r})=0$ and $\boldsymbol{J}^{+}(\mathbf{r})=0$ inside $V$, the Lorentz theorem gives [29]

$$
\begin{aligned}
& \iiint_{V} \nabla \cdot\left[\mathbf{E}^{(\mathrm{tot})-}(\tilde{\mathbf{k}}, \mathbf{r}) \times \mathbf{H}^{(\mathrm{tot})+}\left(\mathbf{h}^{\prime}, \mathbf{r}\right)-\mathbf{E}^{(\mathrm{tot})+}\left(\mathbf{h}^{\prime}, \mathbf{r}\right) \times \mathbf{H}^{(\mathrm{tot})-}(\tilde{\mathbf{k}}, \mathbf{r})\right] d V \\
& \quad=\iiint_{V}\left[\mathbf{E}^{(\mathrm{tot})-}(\tilde{\mathbf{k}}, \mathbf{r}) \cdot \boldsymbol{J}^{+}(\mathbf{r})-\mathbf{E}^{(\mathrm{tot})+}\left(\mathbf{h}^{\prime}, \mathbf{r}\right) \cdot \boldsymbol{J}^{-}(\mathbf{r})\right] d V=0,
\end{aligned}
$$


or, alternatively, using the divergence theorem,

$$
\oiint_{\Sigma}\left[\mathbf{E}^{(\mathrm{tot})-}\left(\tilde{\mathbf{k}}, \mathbf{r}_{0}\right) \times \mathbf{H}^{(\mathrm{tot})+}\left(\mathbf{h}^{\prime}, \mathbf{r}_{0}\right)-\mathbf{E}^{(\mathrm{tot})+}\left(\mathbf{h}^{\prime}, \mathbf{r}_{0}\right) \times \mathbf{H}^{(\mathrm{tot})-}\left(\tilde{\mathbf{k}}, \mathbf{r}_{0}\right)\right] \cdot \hat{\boldsymbol{n}} d S=0,
$$

where $\Sigma$ is the surface enclosing the volume $V$ and $\hat{\boldsymbol{n}}$ a vector normal to this surface at point $\mathbf{r}_{0}=R \hat{\boldsymbol{n}}$. According to the reversibility of Snell's law for refraction, the condition $\mathbf{h}^{\prime}=-\tilde{\mathbf{k}}^{\prime}$ is retained once more. Moreover, reciprocity relations have to be established in the far field so as to make a connection with the optical theorem formulation. For this purpose, the volume $V$ is chosen as a sphere of radius $R$, large enough to have $\mathrm{nk}_{0} R \gg 1$ and allowing the scattered fields to be represented by their asymptotical expressions given in Eqs. (13):

$$
\mathbf{E}^{(\mathrm{scat})-}(\tilde{\mathbf{k}}, \mathbf{r}=R \hat{\boldsymbol{n}})=\Theta(z) \mathbf{A}_{\uparrow}^{-}\left(k_{0} \hat{\boldsymbol{n}}, \tilde{\mathbf{k}}\right) \frac{e^{i k_{0} R}}{R}+\Theta(-z) \mathbf{A}_{\downarrow}^{-}\left(n k_{0} \hat{\boldsymbol{n}}, \tilde{\mathbf{k}}\right) \frac{e^{i n k_{0} R}}{R},
$$

and

$$
\mathbf{E}^{(\mathrm{scat})+}\left(\mathbf{h}^{\prime}=-\tilde{\mathbf{k}}^{\prime}, \mathbf{r}=R \hat{\boldsymbol{n}}\right)=\Theta(z) \mathbf{A}_{\uparrow}^{+}\left(k_{0} \hat{\boldsymbol{n}},-\tilde{\mathbf{k}}^{\prime}\right) \frac{e^{i k_{0} R}}{R}+\Theta(-z) \mathbf{A}_{\downarrow}^{+}\left(n k_{0} \hat{\boldsymbol{n}},-\tilde{\mathbf{k}}^{\prime}\right) \frac{e^{i n k_{0} R}}{R}
$$

where the vector amplitudes are defined in Eq. (14). By making use of the stationary phase method (or Jones' lemma in Appendix XII of Ref. [18]) and exploiting the transversal character of incident and scattered fields, it is possible to establish, from Eq. (34) and using the notations in Eq. (4), the simple relation

$$
k_{z 1}\left[\mathbf{e}^{-}(\tilde{\mathbf{k}}) \cdot \mathbf{e}^{(t)+}\left(-\tilde{\mathbf{k}}^{\prime}\right)\right]-k_{z 2}\left[\mathbf{e}^{(t)-}(\tilde{\mathbf{k}}) \cdot \mathbf{e}^{+}\left(-\tilde{\mathbf{k}}^{\prime}\right)\right]+4 \pi i\left[\mathbf{e}^{+}\left(-\tilde{\mathbf{k}^{\prime}}\right) \cdot \mathbf{A}_{\downarrow}^{-}\left(\tilde{\mathbf{k}^{\prime}}, \tilde{\mathbf{k}}\right)-\mathbf{e}^{-}(\tilde{\mathbf{k}}) \cdot \mathbf{A}_{\uparrow}^{+}\left(-\tilde{\mathbf{k}},-\tilde{\mathbf{k}^{\prime}}\right)\right]=0 .
$$

Since this expression must be valid for any scatterer, the two basic conditions

$$
k_{z 1}\left[\mathbf{e}^{-}(\tilde{\mathbf{k}}) \cdot \mathbf{e}^{(t)+}(-\tilde{\mathbf{k}})\right]=k_{z 2}\left[\mathbf{e}^{(t)-}\left(\tilde{\mathbf{k}^{\prime}}\right) \cdot \mathbf{e}^{+}\left(-\tilde{\mathbf{k}^{\prime}}\right)\right],
$$

and

$$
\mathbf{e}^{+}\left(-\tilde{\mathbf{k}}^{\prime}\right) \cdot \mathbf{A}_{\downarrow}^{-}\left(\tilde{\mathbf{k}^{\prime}}, \tilde{\mathbf{k}}\right)=\mathbf{e}^{-}(\tilde{\mathbf{k}}) \cdot \mathbf{A}_{\uparrow}^{+}\left(-\tilde{\mathbf{k}},-\tilde{\mathbf{k}^{\prime}}\right)
$$

must be fulfilled independently.

\section{Transmission through the interface}

Let us consider first the reciprocity relation (35a) which only concerns the transmission of a plane wave through a planar dielectric interface in the absence of a scatterer. For waves at oblique incidence, the transmitted electric fields are usually expressed in a TE-TM basis set and written as $\mathbf{e}^{(t)+}(-\tilde{\mathbf{k}})=$ $\widehat{T^{\prime}}\left(-\tilde{\mathbf{k}},-\widetilde{\mathbf{k}^{\prime}}\right) \mathbf{e}^{+}\left(-\widetilde{\mathbf{k}}^{\prime}\right)$ and $\mathbf{e}^{(t)-}\left(\widetilde{\mathbf{k}^{\prime}}\right)=\hat{T}\left(\tilde{\mathbf{k}^{\prime}}, \tilde{\mathbf{k}}\right) \mathbf{e}^{-}(\tilde{\mathbf{k}})$ with the transmission operators defined in Eqs. (8c) and (8d). Thanks to the general property $(\hat{U} \mathbf{A}) \cdot(\hat{V} \mathbf{B})=\left(\hat{V}^{\mathrm{T}} \hat{U} \mathbf{A}\right) \cdot \mathbf{B}$, it is straightforward to see that the condition required to fulfill Eq. (35a) is $\widehat{T^{\prime}}\left(-\tilde{\mathbf{k}},-\tilde{\mathbf{k}}^{\prime}\right)=\frac{k_{z 2}}{k_{z 1}}\left[\hat{T}\left(\tilde{\mathbf{k}^{\prime}}, \tilde{\mathbf{k}}\right)\right]^{\mathrm{T}}$, which is nothing but the reciprocity relation [Eq. (26)] established in the previous section. The principle of reciprocity then leads to the laws for the transmission of a plane wave through a diopter, insofar as the materials considered here are passive and linear.
This result obtained for a single interface can be extended to any stratified medium (SF) where the Snell-Descartes laws applied to the multipath transmission through parallel interfaces from air to air impose the conservation of wave vectors $-\tilde{\mathbf{k}}^{\prime}=-\tilde{\mathbf{k}}\left(k_{z 1}=k_{z 2}\right)$ and the TM or TE polarization states (see Fig. 1). The relation (26) reduces to the simplified form $\hat{T}^{\prime S F}(-\tilde{\mathbf{k}},-\tilde{\mathbf{k}})=\left[\hat{T}^{\mathrm{SF}}(\tilde{\mathbf{k}}, \tilde{\mathbf{k}})\right]^{T}$ which prescribes that $t_{s}^{\prime \mathrm{SF}}\left(k_{\|}\right)=t_{s}^{\mathrm{SF}}\left(k_{\|}\right)$and $t_{p}^{\prime}{ }_{p} \mathrm{SF}\left(k_{\|}\right)=t_{p}^{\mathrm{SF}}\left(k_{\|}\right)$and gives the well-known condition fulfilled by the transmission matrices of counter-propagating waves in the Jones' formulation in the case of a normal incidence [16,30].

\section{Scatterer extinction in transmission}

The issue is to discuss, on the basis of relations (35a) and (35b), under which conditions the quantities $\mathrm{C}_{\uparrow}^{(e x t)+}$ and $\mathrm{C}_{\downarrow}^{(\text {ext })-}$ may be equal, or in other terms, Eq. (28a) established in Sec. III A 1 may be verified. Using Eq. (26) and considering that $k_{z 1}$ and $k_{z 2}$ are real-valued, Eq. (28a) gives

$$
\frac{1}{\left|\mathbf{e}^{-}(\tilde{\mathbf{k}})\right|^{2}} \operatorname{Im}\left\{\left[\hat{T}\left(\tilde{\mathbf{k}}^{\prime}, \tilde{\mathbf{k}}\right) \mathbf{e}^{-}(\tilde{\mathbf{k}})\right]^{*} \cdot \mathbf{A}_{\downarrow}^{-}\left(\tilde{\mathbf{k}}^{\prime}, \tilde{\mathbf{k}}\right)\right\}=\frac{1}{\left|\mathbf{e}^{+}\left(-\tilde{\mathbf{k}}^{\prime}\right)\right|^{2}} \operatorname{Im}\left\{\left[\hat{T}\left(\tilde{\mathbf{k}^{\prime}}, \tilde{\mathbf{k}}\right)^{T} \mathbf{e}^{+}\left(-\tilde{\mathbf{k}}^{\prime}\right)\right]^{*} \cdot \mathbf{A}_{\uparrow}^{+}\left(-\tilde{\mathbf{k}},-\widetilde{\mathbf{k}}^{\prime}\right)\right\} .
$$

As before, if one considers that this equality should be also valid for the real parts of the complex scalar products into brackets, it gives

$$
\frac{1}{\left|\mathbf{e}^{-}(\tilde{\mathbf{k}})\right|^{2}}\left\{\left[\hat{T}\left(\tilde{\mathbf{k}}^{\prime}, \tilde{\mathbf{k}}\right) \mathbf{e}^{-}(\tilde{\mathbf{k}})\right]^{*} \cdot \mathbf{A}_{\downarrow}^{-}\left(\widetilde{\mathbf{k}^{\prime}}, \tilde{\mathbf{k}}\right)\right\}=\frac{1}{\left|\mathbf{e}^{+}\left(-\widetilde{\mathbf{k}}^{\prime}\right)\right|^{2}}\left\{\left[\hat{T}\left(\tilde{\mathbf{k}^{\prime}}, \tilde{\mathbf{k}}\right)^{T} \mathbf{e}^{+}\left(-\widetilde{\mathbf{k}}^{\prime}\right)\right]^{*} \cdot \mathbf{A}_{\uparrow}^{+}\left(-\tilde{\mathbf{k}},-\widetilde{\mathbf{k}^{\prime}}\right)\right\}
$$


A direct comparison between Eq. (37) and relation (35b) leads to the same relation as Eq. (29) established in Sec. III A 1, and therefore to the same general conclusion. The insensitivity of the extinction cross section of the transmitted wave to a reversal of the excitation scheme requires the commutation condition $\left[\hat{T}\left(\tilde{\mathbf{k}}^{\prime}, \tilde{\mathbf{k}}\right)^{\mathrm{T} *} \hat{T}\left(\tilde{\mathbf{k}}^{\prime}, \tilde{\mathbf{k}}\right), \hat{I}(\tilde{\mathbf{k}}, \tilde{\mathbf{k}})\right]=0$. This property can be fulfilled in the case of a normal incidence or an oblique incidence with pure TE or TM polarizations, with the same constraint on the relative polarization of both counter-propagating waves. However, unlike the Green's function formalism, nothing more can be said about the sensitivity of the extinction of the reflected wave relative to a reversal of the excitation scheme.

\section{DISCUSSION}

In many cases of interest, the extinction cross section of the light transmitted through an interface in the presence of a scatterer is found to be strictly insensitive to the reversal of the excitation scheme, primarily if the transmitted wave preserves the polarization state of the incident wave. This property finds its origin in the special symmetry of the Green's operator $\left[\hat{G}\left(\mathbf{r}, \mathbf{r}^{\prime}\right)=\hat{G}\left(\mathbf{r}^{\prime}, \mathbf{r}\right)^{\mathrm{T}}\right]$ and was established through two basically equivalent approaches (Green's formalism or the Lorentz-Helmholtz theorem). It is strictly valid in the case of normal incidence or in the case of oblique incidence for purely TE or TM polarized waves, and only approximate otherwise, as discussed hereafter. Such a property is not so intuitive even in the absence of a dielectric interface (free scatterer in a homogeneous medium) where the total scattering (or absorption) cross section itself is in general not invariant. It is intimately related to the cancellation of retardation effects through the scatterer volume, i.e., the conservation of phase coherence between the local excitation field and the far fields scattered by elementary parts of the scatterer in the same propagation direction. Such a coherence is destroyed in the case of the reflected wave because of mirroring and induced retardation effects. This is obvious in the expression of the backscattering operators $\hat{S}_{\uparrow}^{-}(\mathbf{k}, \tilde{\mathbf{k}})$ and $\hat{S}_{\downarrow}^{+}\left(-\mathbf{k}^{\prime},-\tilde{\mathbf{k}}^{\prime}\right)$ in Eqs. (33), where transmission and reflection coefficients play asymmetric roles.

\section{A. Normal incidence with circularly polarized waves}

Normal incidence is an important case encountered in most experimental studies, with especially profound implications on circular dichroism properties. In this respect, clear conventions for the description of polarization states of the incident waves relative to their direction of propagation are required. The main specificity of this configuration is that the positively oriented basis frames attached to the descending and ascending waves $(\hat{\boldsymbol{s}}(\tilde{\mathbf{k}}), \hat{\boldsymbol{p}}(\tilde{\mathbf{k}}), \widehat{\tilde{\mathbf{k}}})$ and $\left(\hat{\boldsymbol{s}}\left(-\tilde{\mathbf{k}}^{\prime}\right)\right.$, $\left.\hat{\boldsymbol{p}}\left(-\tilde{\mathbf{k}}^{\prime}\right),-\widehat{\tilde{\mathbf{k}}^{\prime}}\right)$, respectively, are parallel to each other. At the limit of normal incidence $\left(k_{\|} \rightarrow 0\right)$, the TM-TE basis vectors coincide with the axes of the laboratory frame with the following relations, $\widehat{\tilde{\mathbf{k}}} \rightarrow-\hat{\mathbf{z}},-\widehat{\tilde{\mathbf{k}}^{\prime}} \rightarrow \hat{\mathbf{z}}, \hat{s}(\tilde{\mathbf{k}}) \rightarrow$ $\hat{\boldsymbol{y}}, \hat{p}(\tilde{\mathbf{k}}) \rightarrow \hat{\boldsymbol{x}}, \hat{s}\left(-\tilde{\mathbf{k}}^{\prime}\right) \rightarrow-\hat{\boldsymbol{y}}$, and $\hat{p}\left(-\tilde{\mathbf{k}}^{\prime}\right) \rightarrow \hat{\boldsymbol{x}}$ (Fig. 7). The relation $e_{s}^{+}\left(-\tilde{\mathbf{k}}^{\prime}\right) / e_{p}^{+}\left(-\tilde{\mathbf{k}}^{\prime}\right)=-\left[e_{s}^{-}(\tilde{\mathbf{k}}) / e_{p}^{-}(\tilde{\mathbf{k}})\right]^{*}$ between the components of the electric-field polarizations results from the

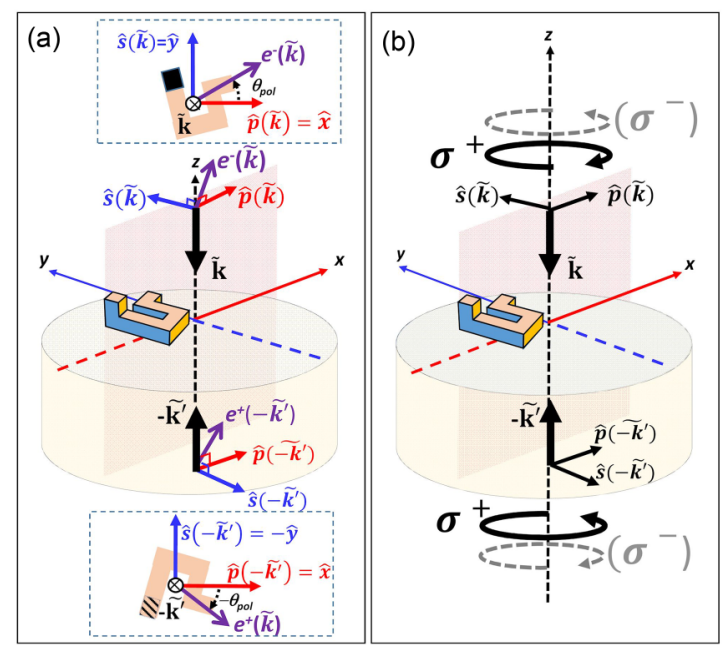

FIG. 7. Relationships between the polarization states of counterpropagating waves exciting a supported scatterer at normal incidence for achieving invariance in the extinction cross section for transmitted waves. (a) Case of a linear polarization, the insets show the orientation of the polarization vectors in the plane normal to the wave direction of propagation with the wave vector seen from the rear, together with the corresponding view of the supported scatterer. (b) Case of circularly polarized waves, the handedness of counterpropagating waves must be the same, either positive (black arrow) or negative (gray dotted arrow).

constraint $\mathbf{e}^{+}\left(-\tilde{\mathbf{k}}^{\prime}\right) /\left|\mathbf{e}^{+}\left(-\tilde{\mathbf{k}}^{\prime}\right)\right|=\mathbf{e}^{-}(\tilde{\mathbf{k}})^{*} /\left|\mathbf{e}^{-}(\tilde{\mathbf{k}})\right|$ on their amplitudes [see Eq. (30) with $\beta=0$ ] because $\hat{\boldsymbol{s}}\left(-\tilde{\mathbf{k}}^{\prime}\right)=-\hat{\boldsymbol{s}}(\tilde{\mathbf{k}})$ and $\hat{\boldsymbol{p}}\left(-\tilde{\mathbf{k}}^{\prime}\right)=\hat{\boldsymbol{p}}(\tilde{\mathbf{k}})$. If the wave coming from the upper half space is linearly polarized $\left[e_{s}^{-}(\tilde{\mathbf{k}}) / e_{p}^{-}(\tilde{\mathbf{k}})=\tan \left(\theta_{\mathrm{pol}}\right)\right]$, then the wave coming from the lower half space must have the same polarization but with an apparent angle of opposite sign $\left[e_{s}^{+}\left(-\tilde{\mathbf{k}}^{\prime}\right) / e_{p}^{+}\left(-\tilde{\mathbf{k}}^{\prime}\right)=\tan \left(-\theta_{\mathrm{pol}}\right)\right]$, as long as the object and the oriented frames are seen from the far source regions, the wave vectors $\tilde{\mathbf{k}}$ and $-\tilde{\mathbf{k}}^{\prime}$ pointing towards opposite directions [Fig. 7(a)].

In the case of circularly polarized waves, a right-handed (positive) or a left-handed (negative) circular polarization is, by convention here, associated with a clockwise or a counterclockwise rotation of the electric field relative to the direction of propagation when observed from the corresponding source region. Owing to the choice of the temporal dependence $\left(e^{-i \omega t}\right)$, normalized positive or negative circularly incident waves coming from the upper half space are written $\mathbf{e}^{-}(\tilde{\mathbf{k}})=$ $\hat{\boldsymbol{\sigma}}^{ \pm}(\tilde{\mathbf{k}})=\frac{1}{\sqrt{2}}[\hat{\boldsymbol{s}}(\tilde{\mathbf{k}}) \mp i \hat{\boldsymbol{p}}(\tilde{\mathbf{k}})]$. To achieve invariance of the extinction cross sections of the transmitted waves relative to a reversal of the excitation scheme, the constraint (30) imposes the polarization states of the waves coming from the lower half space to be written as $\mathbf{e}^{+}\left(-\tilde{\mathbf{k}}^{\prime}\right)=\frac{1}{\sqrt{2}}[\hat{\boldsymbol{s}}(\tilde{\mathbf{k}}) \mp i \hat{\boldsymbol{p}}(\tilde{\mathbf{k}})]^{*}=$ $\frac{1}{\sqrt{2}}\left[-\hat{\boldsymbol{s}}\left(-\tilde{\mathbf{k}}^{\prime}\right) \pm i \hat{\boldsymbol{p}}\left(-\tilde{\mathbf{k}}^{\prime}\right)\right]=-\hat{\boldsymbol{\sigma}}^{ \pm}\left(-\tilde{\mathbf{k}}^{\prime}\right)$ and therefore must possess the same handedness [Fig. 7(b)].

An important consequence of this property is illustrated in Fig. 8, which shows the particular example of a scatterer consisting of a spiral shined on by a positive circularly plane wave directed normally to its mirror plane of symmetry. This spiral is achiral since it can be superposed to its mirror image through a suited rotation but, viewed in the direction 


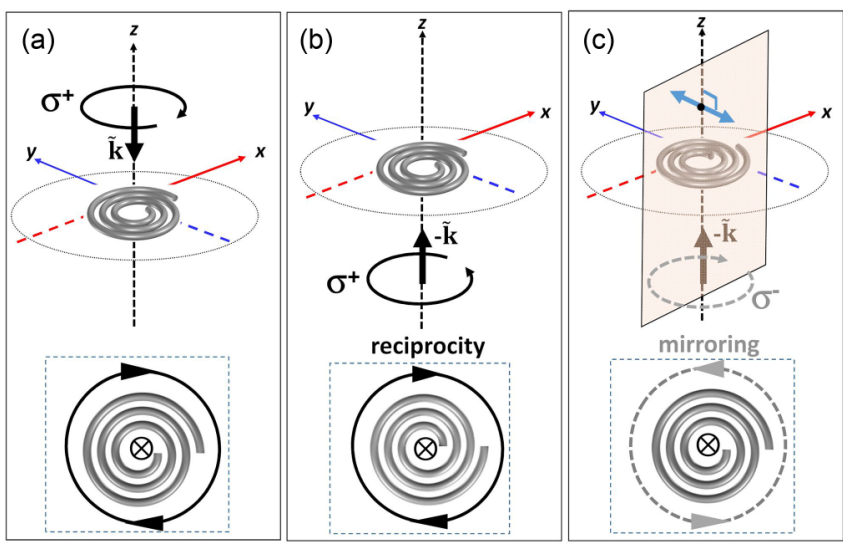

FIG. 8. (a) Spiral embedded in a homogeneous medium and excited by a circularly polarized plane wave normally incident relative to its plane of symmetry. The wave and the spiral are both righthanded. (b) Excitation scheme of the same spiral by a circularly polarized plane wave propagating in the opposite direction and retaining the same handedness. (c) Mirror transformation of scheme (b) relative to a plane containing the incident wave vector. The circular polarization turns from positive to negative (gray dotted arrow).

of the incident wave vector, it possesses a sense of twist chosen to coincide with the handedness of the excitation wave [Fig. 8(a)]. According to the property established before, the extinction cross section is unchanged if the spiral is excited by a positive circularly plane wave propagating in the opposite direction, although the apparent sense of twist of the spiral seen from the light source regions is reversed [Fig. 8(b)]. Starting from this second configuration, a mirror inversion relative to a plane normal to the spiral would result in changing the handedness of both the spiral and the wave polarization without altering the incident wave vector direction [Fig. 8(c)]. In such an operation, the extinction cross section is unchanged. As a consequence, a comparison between the configurations in Figs. 8(a) and 8(c) proves the absence of circular dichroism with respect to extinction measurements for such a planar object. This does not preclude its occurrence for absorption and total scattering.

\section{B. Failure of the invariance of the extinction cross section for reversed excitation schemes in the general case: Numerical verifications}

In case of an arbitrary polarized wave at oblique incidence, the extinction of the transmitted light depends on whether the supported scatterer is illuminated from the upper or from the lower medium because the operators $\hat{T}\left(\tilde{\mathbf{k}}^{\prime}, \tilde{\mathbf{k}}\right)^{\mathrm{T} *} \hat{T}\left(\tilde{\mathbf{k}}^{\prime}, \tilde{\mathbf{k}}\right)$ and $\hat{I}(\tilde{\mathbf{k}}, \tilde{\mathbf{k}})$ do not commute due to the difference between $\mathrm{TE}$ and TM transmission coefficients $\left[t_{p}\left(k_{\|}\right)\right.$and $\left.t_{s}\left(k_{\|}\right)\right]$. In order to highlight and quantify this phenomenon, extinction cross sections given by Eqs. (22a) and (23a) are calculated in the specific case of a scatterer consisting of a gold plasmonic structure chosen for its generality, without any particular mirror symmetry (a priori chiral), placed in air and supported on a plane dielectric semi-infinite substrate (refractive index $n$ ) as shown in Fig. 9. The plane of incidence for the excitation waves is chosen as the $x z$ plane in the laboratory frame. The polar angles of incidence for the plane waves coming from the air $(\theta)$ or from the dielectric $(\varphi)$ are related by Snell's laws $\left(\sin \theta=n \sin \varphi, \cos \theta=\frac{k_{z 1}}{k_{0}}\right.$ and $\cos \varphi=\frac{k_{z 2}}{n k_{0}}$ ).

In a first step, Maxwell's equations are solved in the nearfield by using a finite-element method (COMSOL 5.2 software). The dielectric function of gold is taken from Ref. [31]. The calculation parameters are the angle of incidence $\theta$ and the polarization of the incident fields $\mathbf{e}^{-}(\tilde{\mathbf{k}})=\mathrm{e}_{s}^{-}(\tilde{\mathbf{k}}) \hat{\boldsymbol{s}}(\tilde{\mathbf{k}})+$ $e_{p}^{-}(\tilde{\mathbf{k}}) \hat{\boldsymbol{p}}(\tilde{\mathbf{k}})$ and $\mathbf{e}^{+}\left(-\tilde{\mathbf{k}}^{\prime}\right)=e_{s}^{+}\left(-\tilde{\mathbf{k}}^{\prime}\right) \hat{\boldsymbol{s}}\left(-\tilde{\mathbf{k}}^{\prime}\right)+e_{p}^{+}\left(-\tilde{\mathbf{k}}^{\prime}\right) \hat{\boldsymbol{p}}\left(-\tilde{\mathbf{k}}^{\prime}\right)$ (see Fig. 9) that are assumed to be linear $\left[e_{s}^{-}(\tilde{\mathbf{k}}), e_{p}^{-}(\tilde{\mathbf{k}})\right.$, $e_{s}^{+}\left(-\tilde{\mathbf{k}}^{\prime}\right)$, and $e_{p}^{+}\left(-\tilde{\mathbf{k}}^{\prime}\right)$ are real]. The polarization angle $\theta_{\mathrm{pol}}$ of the wave coming from the upper space (air) is defined through the ratio $e_{p}^{-}(\tilde{\mathbf{k}}) / e_{s}^{-}(\tilde{\mathbf{k}})=\tan \left(\theta_{\mathrm{pol}}\right)$. For an oblique incidence, there is no longer preferred relations between the polarizations of the incident fields $\mathbf{e}^{-}(\tilde{\mathbf{k}})$ and $\mathbf{e}^{+}\left(-\tilde{\mathbf{k}}^{\prime}\right)$. One could choose $\mathbf{e}^{+}\left(-\tilde{\mathbf{k}}^{\prime}\right)$ proportional to the field transmitted from the air to the substrate $\left[\hat{T}\left(\tilde{\mathbf{k}}^{\prime}, \tilde{\mathbf{k}}\right) \mathbf{e}^{-}(\tilde{\mathbf{k}})\right]$ or, reciprocally, choose $\mathbf{e}^{+}\left(-\tilde{\mathbf{k}}^{\prime}\right)$ such as the field transmitted from the substrate to the air
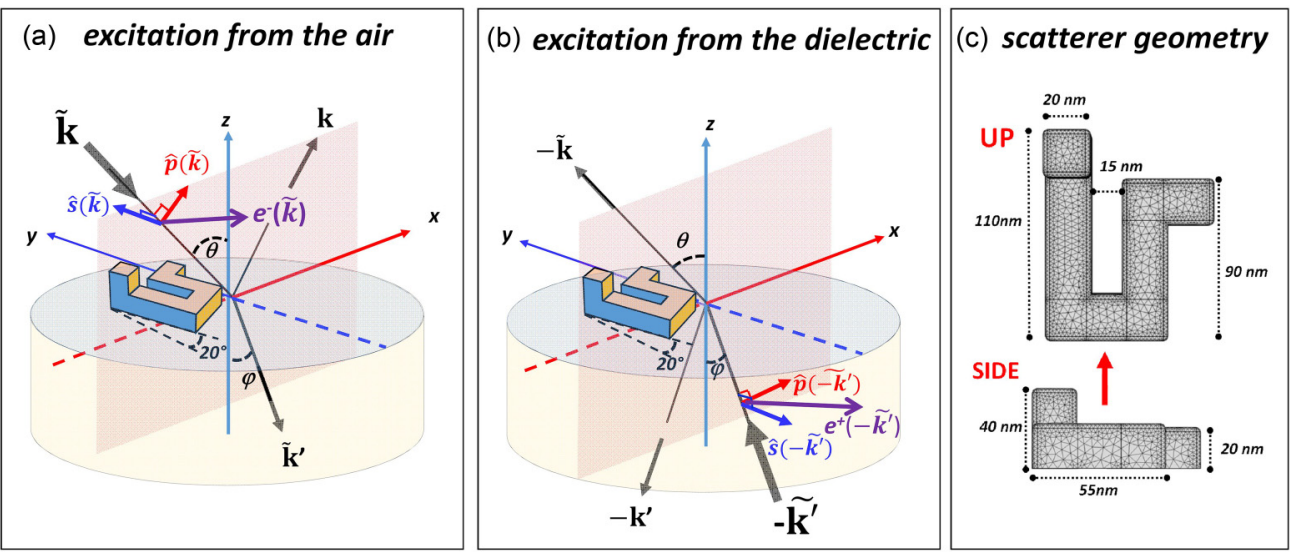

FIG. 9. Schemes of the excitation configurations involved in the numerical calculations. The incident wave can come (a) from the air with an angle of incidence $\theta$ in the $x z$ plane or (b) from the substrate of refractive index $n$ with an angle of incidence $\varphi=\sin { }^{-1}(\sin \theta / n)$ in the $x z$ plane (c) The long side of the gold nanostructure forms an angle of $20^{\circ}$ relative to the $y$ axis. 

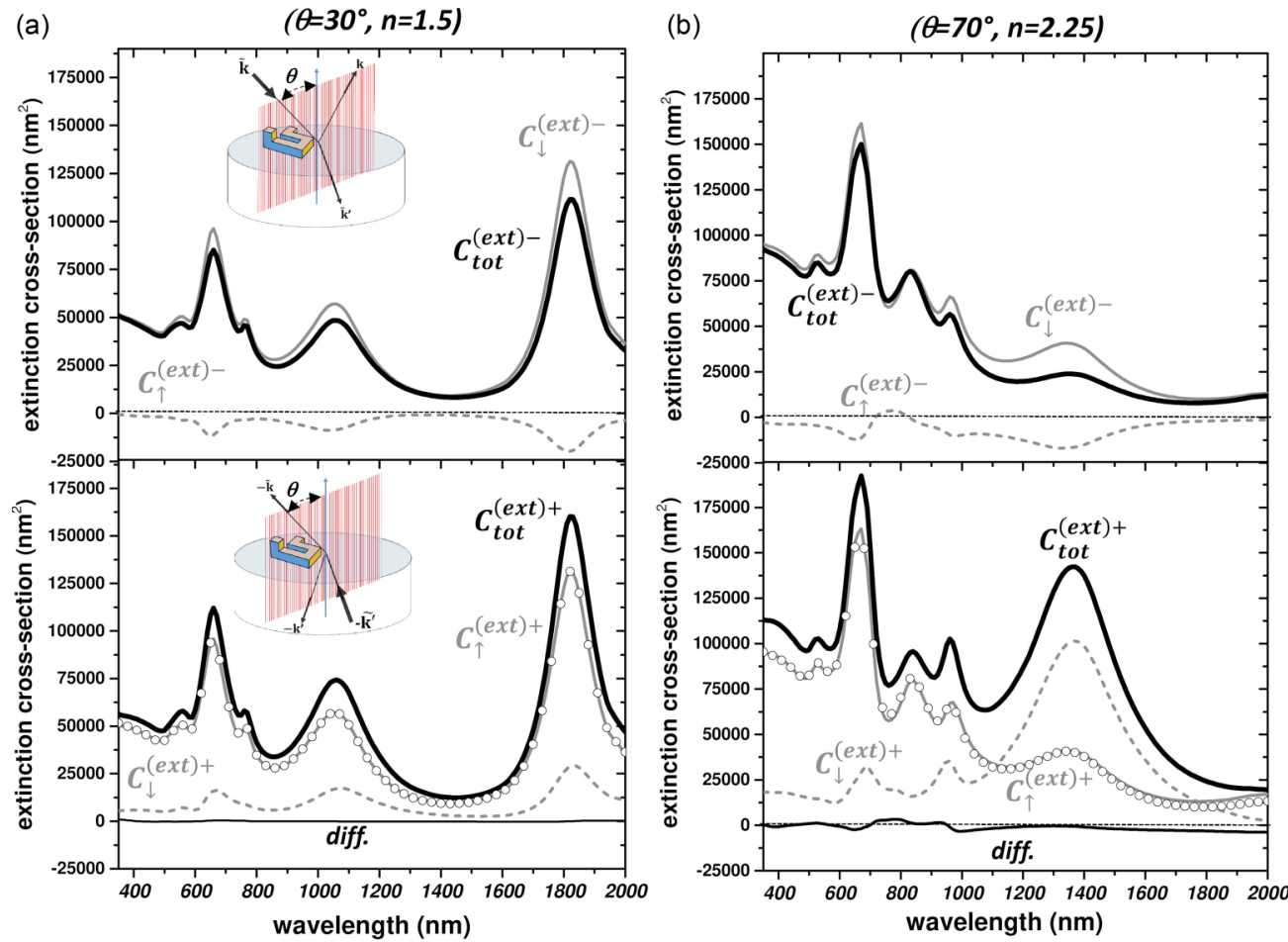

FIG. 10. Extinction cross sections for the transmitted (full gray lines) and reflected (dotted gray lines) waves calculated in the case of an excitation of the supported gold nanostructure from the air (upper panels) or from the dielectric (lower panels). Their sums (total extinctions) are given by the thick black lines. The wave coming from the upper half space is linearly polarized with an angle $\theta_{\mathrm{pol}}=60^{\circ}$. Calculations are made for an angle of incidence $\theta=30^{\circ}$ and a refractive index of the substrate $n=1.5$ in panel (a) and for $\theta=70^{\circ}$ and $n=2.25$ in panel (b). In the lower panels, the circles report the values of $C_{\downarrow}^{(\text {ext })-}$ from the upper panel for comparison with $C_{\uparrow}^{(\text {ext)+ }}$. Their difference is given by the lowermost thick black curves.

$\left[\widehat{T}^{\prime}\left(-\tilde{\mathbf{k}},-\tilde{\mathbf{k}}^{\prime}\right) \mathbf{e}^{+}\left(-\tilde{\mathbf{k}}^{\prime}\right)\right]$ is proportional to $\mathbf{e}^{-}(\tilde{\mathbf{k}})$. None of these nonequivalent hypotheses is more relevant than the other. For simplicity and by analogy with the limiting case of a normal incidence, we arbitrarily assume that $\frac{e_{s}^{+}\left(-\tilde{\mathbf{k}}^{\prime}\right)}{e_{p}^{+}\left(-\tilde{\mathbf{k}}^{\prime}\right)}=\tan \left(-\theta_{\mathrm{pol}}\right)$ which is also consistent with the case of pure TE or TM polarizations at oblique incidence. In a second step, the nearfield to far-field transformation is computed by using a method based on reciprocity arguments and a freely available software package [32].

Figure 10(a) shows that, for the choice $\theta=30^{\circ}, \theta_{\text {pol }}=$ $60^{\circ}$, and $n=1.5$, the cross sections for the reflected waves are totally different for the two directions of excitation and may adopt negative values corresponding to an increase of the reflected power. On the contrary, the curves $C_{\downarrow}^{\text {(ext)- }}$ and $C_{\uparrow}^{(\text {ext)+ }}$ cannot be distinguished from each other in the whole spectral range where several plasmon resonances develop. This is due here to the close proximity between the TE and TM transmission coefficients $\left(t_{s}\left(k_{\|}\right) / t_{p}\left(k_{\|}\right) \simeq 0.982\right.$ for $\theta=30^{\circ}$ and $n=1.5$ as shown in Fig. 11 of Appendix A. If the angle of incidence and/or the refractive index of the substrate is increased, the difference between the transmission coefficients for TE and TM polarizations become larger, leading to a weak but noticeable dependence of the extinction of the transmitted waves on the excitation scheme, as illustrated in Fig. 10(b) for $\theta=70^{\circ}$ and $n=2.25$, corresponding to $t_{s}\left(k_{\|}\right) / t_{p}\left(k_{\|}\right) \simeq 0.74$. It must be noted that such extreme parameter values are not commonly encountered in most optical experiments. In any case, the sums $C_{\text {tot }}^{(\text {ext)- }}=C_{\uparrow}^{(\text {ext })-}+$ $C_{\downarrow}^{(\mathrm{ext})-}$ and $C_{\mathrm{tot}}^{(\mathrm{ext})+}=C_{\uparrow}^{(\mathrm{ext})+}+C_{\downarrow}^{(\mathrm{ext})+}$ corresponding to the total extinction cross sections are significantly different for reversed-excitation schemes.

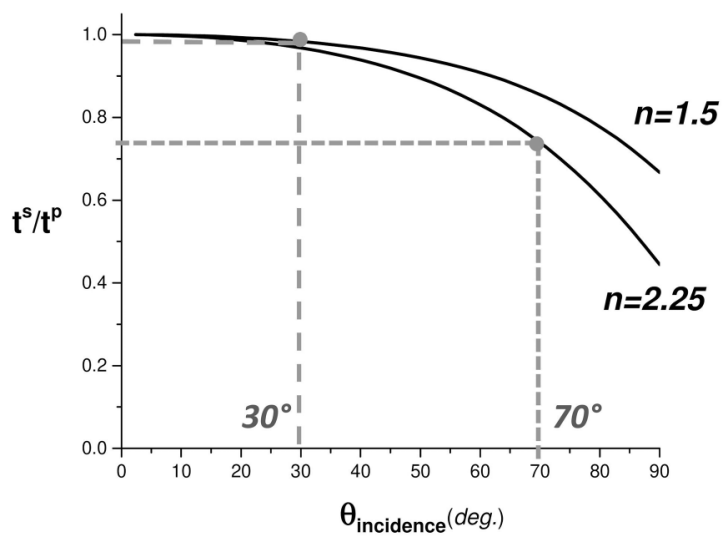

FIG. 11. Ratio between the TE and TM transmission coefficients from air to a dielectric medium of refractive index $n=1.5$ or $n=$ 2.25 as a function of the angle of incidence. The values $\approx 0.74$ for $\theta=70^{\circ}(n=2.25)$ and $\approx 0.98$ for $\theta=30^{\circ}(n=1.5)$ are indicated. 


\section{CONCLUSION}

We have analyzed to what extent, and under which conditions, the extinction of the transmitted light by a scatterer placed at the planar interface between two isotropic and semiinfinite homogeneous media can be sensitive to the direction under which it may be illuminated. Extinction cross sections in transmission are found to be exactly identical for reversed excitation configurations as far as normal incidence or oblique incidence with purely magnetic or electric transverse excitation waves are considered. This has been demonstrated from two converging approaches: a Green's function integral equation method and an application of the Lorentz reciprocity theorem in optics. The fundamental condition is that the polarization state of an incident excitation wave coming from a given medium must be the complex conjugate of the polarization state of the counter-propagating wave transmitted in the same medium. This amounts to a simple conservation of the linear TE or TM polarization for an oblique incidence and to the conservation of a general elliptical polarization handedness for normal incidence. For an oblique incidence with waves being neither purely TE nor TM linearly polarized, the extinction cross sections for reversed illumination schemes are no longer identical. This deviation is all the more significant that TM and TE transmission coefficients themselves differ from each other (large angle of incidence and/or large refractive index of the dielectric medium), inducing a polarization rotation upon transmission. This fully corroborates the observations recently made in extinction spectroscopy experiments on single plasmonic nanoparticles supported on transparent thin film or thick glass slides [7]. Moreover, except for specific limiting situations, this property does not hold for the extinction of reflected waves and therefore for the global extinction by the supported particle, according to the generalized optical theorem.

This restricting condition on the polarization of reciprocal waves is of main importance with regard to the chiral properties of supported particles. For instance, this shows that, when embedded in a homogeneous medium, any scatterer having a plane of symmetry normal to the incident wave vector cannot display any dichroism in the extinction of transmitted light, even if it may possess a sense of twist. This observation is related to the more general and widely debated issue of the chirality and dichroic properties of two-dimensional metamaterials made of planar nanostructures arranged in regular arrays [33-37]. In this respect, the general connection that can be made between the circular dichroism and/or the optical activity of free-standing or supported scatterers and their symmetry properties will be explored more deeply in a forthcoming presentation that should benefit from the Green's equation integral formalism introduced here.

\section{ACKNOWLEDGMENTS}

The authors are grateful to R. Mascart for the management of computer facilities and especially thank M. Broyer and E. Cottancin for fruitful discussions and comments. They acknowledge support by the French region Rhône Alpes Auvergne (Optolyse, CPER2016) and their funding institutions: Centre National de la Recherche Scientifique and Ministère de l'Enseignement Supérieur, de la Recherche et de l'Innovation.

\section{APPENDIX A: THE FRESNEL COEFFICIENTS}

For a plane wave incident on a planar interface between two media 1 and 2 with dielectric functions $\varepsilon_{1}$ and $\varepsilon_{2}$ and magnetic permittivities $\mu_{1}$ and $\mu_{2}$ as sketched in Fig. 3, and with the convention chosen for the TE (s) and TM (p) basis [Eqs. (6) and (7)], the general reflection and transmission coefficients are written [21]

$$
\begin{array}{ll}
r_{s}^{1 \rightarrow 2}\left(k_{x}, k_{y}\right)=\frac{\mu_{2} k_{z 1}-\mu_{1} k_{z 2}}{\mu_{2} k_{z 1}+\mu_{1} k_{z 2}}, & r_{p}^{1 \rightarrow 2}\left(k_{x}, k_{y}\right)=\frac{\varepsilon_{2} k_{z 1}-\varepsilon_{1} k_{z 2}}{\varepsilon_{2} k_{z 1}+\varepsilon_{1} k_{z 2}}, \\
t_{s}^{1 \rightarrow 2}\left(k_{x}, k_{y}\right)=\frac{2 \mu_{2} k_{z 1}}{\mu_{2} k_{z 1}+\mu_{1} k_{z 2}}, & t_{p}^{1 \rightarrow 2}\left(k_{x}, k_{y}\right)=\frac{2 \varepsilon_{2} k_{z 1}}{\varepsilon_{2} k_{z 1}+\varepsilon_{1} k_{z 2}} \sqrt{\frac{\mu_{2} \varepsilon_{1}}{\mu_{1} \varepsilon_{2}}},
\end{array}
$$

with $k_{z 1}$ and $k_{z 2}$ defined in Eq. (2). The superscript $(1 \rightarrow 2)$ indicates that the wave propagates from medium1 to medium 2 . In the case of a plane wave incident from medium 2 to medium 1, it is straightforward to check that $t_{(s / p)}^{2 \rightarrow 1}\left(k_{x}, k_{y}\right)=\frac{k_{z 2} \mu_{1}}{k_{z 1} \mu_{2}} t_{(s / p)}^{1 \rightarrow 2}\left(k_{x}, k_{y}\right)$ and $r_{(s / p)}^{2 \rightarrow 1}\left(k_{x}, k_{y}\right)=-r_{(s / p)}^{1 \rightarrow 2}\left(k_{x}, k_{y}\right)$. Figure 11 shows the dependence with the angle of incidence of the ratio between the transmission coefficients of TM and TE waves, when medium 1 is the air and medium 2 is a dielectric of refractive index $n=1.5$ or 2.25

\section{APPENDIX B: EXPRESSIONS OF THE SCATTERING VECTOR AMPLITUDES}

We derive here the particular expression (14a) of the scattering vector amplitude $\mathbf{A}_{\downarrow}^{-}\left(\tilde{\mathbf{K}}^{\prime}, \tilde{\mathbf{k}}\right)=$ $-\mathrm{i} 2 \mathrm{~K}_{z}^{\prime} e^{\mathrm{i} \mathrm{K}_{z}^{\prime} \cdot z_{b}} \iint_{z=z_{b}} d^{2} r_{\|} e^{-i \tilde{\mathbf{K}}^{\prime} \cdot \mathbf{r}_{\|}} \mathbf{E}^{(\text {scat })-}(\tilde{\mathbf{k}}, \mathbf{r})$ defined as the Fourier component [Eq. (13b)] of the scattered field $\mathbf{E}^{\text {(scat })-}(\tilde{\mathbf{k}}, \mathbf{r})=k_{0}^{2} \iiint_{\mathrm{Vs}_{\mathrm{s}}} d^{3} r^{\prime} \eta\left(\mathbf{r}^{\prime}\right) \hat{G}\left(\mathbf{r}, \mathbf{r}^{\prime}\right) \mathbf{E}^{(\text {tot })-}\left(\tilde{\mathbf{k}}, \mathbf{r}^{\prime}\right)$ [Eq. (11)]. The arbitrary coordinate $z_{b}<0$ on the $z$ axis is chosen so that the scatterer is entirely located above the corresponding plane parallel to the interface [see Fig. 3(a)]. In that case, the Green's operator $\hat{G}\left(\mathbf{r}, \mathbf{r}^{\prime}\right)[$ Eq. (12)] is written

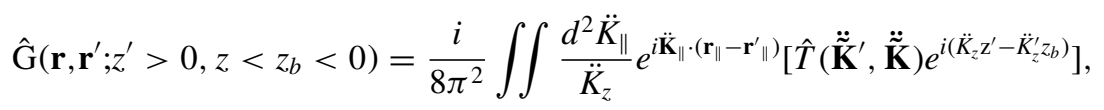


since the scatterer lies in the upper half space $\left(z^{\prime}>0\right)$ and the scattered field is considered in the region where $\mathrm{z}<z_{b}<0$. The variable $\ddot{\mathbf{K}}$ is used instead of $\mathbf{K}$ to avoid confusion with the argument $\tilde{\mathbf{K}}^{\prime}$ in $\mathbf{A}_{\downarrow}^{-}\left(\tilde{\mathbf{K}}^{\prime}, \tilde{\mathbf{k}}\right)$. Therefore,

$$
\begin{aligned}
& \mathbf{A}_{\downarrow}^{-}\left(\tilde{\mathbf{K}}^{\prime}, \tilde{\mathbf{k}}\right)=-\mathrm{i} 2 \mathbf{K}_{z}^{\prime} e^{i K_{z}^{\prime} \cdot z_{b}} k_{0}^{2} \iint_{z=z_{b}} d^{2} r_{\|} e^{-i \tilde{\mathbf{K}}^{\prime} \cdot \mathbf{r}_{\|}} \iiint_{V_{S}} d^{3} r^{\prime}\left\{\frac{i}{8 \pi^{2}} \iint \frac{d^{2} \ddot{K}_{\|}}{\ddot{K}_{z}} e^{i \ddot{\mathbf{K}}_{\|} \cdot\left(\mathbf{r}_{\|}-\mathbf{r}_{\|}^{\prime}\right)}\left[\hat{T}\left(\ddot{\tilde{\mathbf{K}}}^{\prime}, \ddot{\tilde{\mathbf{K}}}\right) e^{i\left(\ddot{K}_{z} z^{\prime}-\ddot{K}_{z}^{\prime} z_{b}\right)}\right]\right\} \eta\left(\mathbf{r}^{\prime}\right) \mathbf{E}^{(\mathrm{tot})-}\left(\tilde{\mathbf{k}}, \mathbf{r}^{\prime}\right) \\
& =-\mathrm{i} 2 \mathbf{K}_{z}^{\prime} e^{i K_{z}^{\prime} \cdot z_{b}} k_{0}^{2} \iiint_{V_{S}} d^{3} r^{\prime} e^{-i \ddot{\mathbf{K}}_{\|} \cdot \mathbf{r}_{\|}^{\prime} \|}\left\{\frac{i}{8 \pi^{2}} \iint \frac{d^{2} \ddot{K}_{\|}}{\ddot{K}_{z}}\left[\iint_{z=z_{b}} d^{2} r_{\|} e^{i\left(\ddot{\mathbf{K}}_{\|}-\tilde{\mathbf{K}}_{\|}^{\prime}\right) \cdot \mathbf{r}_{\|}}\right]\left[\hat{T}\left(\ddot{\tilde{\mathbf{K}}}, \ddot{\tilde{\mathbf{K}}}^{\prime}\right) e^{i\left(\ddot{K}_{z} z^{\prime}-\ddot{K}_{z}^{\prime} z_{b}\right)}\right]\right\} \eta\left(\mathbf{r}^{\prime}\right) \mathbf{E}^{(\mathrm{tot})-}\left(\tilde{\mathbf{k}}, \mathbf{r}^{\prime}\right) .
\end{aligned}
$$

The integration over $d^{2} r_{\|}$in the plane $z=z_{b}$ provides the Dirac delta function $(2 \pi)^{2} \delta^{(2)}\left(\ddot{\mathbf{K}}_{\|}-\mathbf{K}_{\|}\right)$which imposes $\ddot{\mathbf{K}}_{\|}-\mathbf{K}_{\|}=$ $\ddot{\mathbf{K}}_{\|}^{\prime}-\mathbf{K}_{\|}^{\prime}=0$ and then $\ddot{K}_{z}=K_{z}$ and $\ddot{K}_{z}^{\prime}=\mathrm{K}_{z}^{\prime}$ according to Eqs (2). Since $\ddot{\mathbf{K}}_{\|} \cdot \mathbf{r}_{\|}^{\prime}-K_{z} z^{\prime}=\tilde{\mathbf{K}} \cdot \mathbf{r}^{\prime}$, we finally obtain

$$
\begin{aligned}
\mathbf{A}_{\downarrow}^{-}\left(\tilde{\mathbf{K}}^{\prime}, \tilde{\mathbf{k}}\right) & =-\mathrm{i} 2 \mathrm{~K}_{z}^{\prime} e^{\mathrm{i} K_{z}^{\prime} \cdot z_{b}} k_{0}^{2} \iiint_{V_{S}} d^{3} r^{\prime} e^{-i \ddot{\mathbf{K}}_{\|} \cdot \mathbf{r}^{\prime} \|}\left\{\frac{(2 \pi)^{2} i}{8 \pi^{2} K_{z}}\left[\hat{T}\left(\tilde{\mathbf{K}} \tilde{\mathbf{K}}^{\prime}, \mathbf{K}\right) e^{i\left(K_{z} z^{\prime}-K_{z}^{\prime} z b\right)}\right]\right\} \eta\left(\mathbf{r}^{\prime}\right) \mathbf{E}^{(\mathrm{tot})-}\left(\tilde{\mathbf{k}}, \mathbf{r}^{\prime}\right) \\
& =\frac{K_{z}^{\prime}}{K_{z}} k_{0}^{2} \iiint_{V_{S}} d^{3} r^{\prime} e^{-i \tilde{\mathbf{K}} \cdot \mathbf{r}^{\prime}} \hat{T}\left(\tilde{\mathbf{K}}^{\prime}, \mathbf{K}\right) \eta\left(\mathbf{r}^{\prime}\right) \mathbf{E}^{(\mathrm{tot})-}\left(\tilde{\mathbf{k}}, \mathbf{r}^{\prime}\right) .
\end{aligned}
$$

\section{APPENDIX C: SINGULARITY OF THE GREEN'S FUNCTION IN THE SCATTERER VOLUME}

The integral formulation of the scattered electric field, $\mathbf{E}^{\text {(scat) }}(\mathbf{r})=k_{0}^{2} \iiint_{\mathrm{Vs}_{\mathrm{s}}} d^{3} r^{\prime} \eta\left(\mathbf{r}^{\prime}\right) \hat{G}\left(\mathbf{r}, \mathbf{r}^{\prime}\right)\left[\mathbf{E}^{\text {(inc) }}\left(\mathbf{r}^{\prime}\right)+\mathbf{E}^{\text {(scat) }}\left(\mathbf{r}^{\prime}\right)\right]$, is only valid outside the volume of the scatterer $\left(\mathbf{r} \neq \mathbf{r}^{\prime}\right)$. The Green's function of the problem [Eq. (12)] can be split into two contributions, $\hat{G}\left(\mathbf{r}, \mathbf{r}^{\prime}\right)=\hat{G}_{R}\left(\mathbf{r}, \mathbf{r}^{\prime}\right)+\hat{G}_{0}\left(\mathbf{r}, \mathbf{r}^{\prime}\right)$, where $\hat{G}_{R}\left(\mathbf{r}, \mathbf{r}^{\prime}\right)=\frac{i}{8 \pi^{2}} \iint \frac{d^{2} K_{\|}}{K_{z}} e^{i \mathbf{K}_{\|} \cdot\left(\mathbf{r}_{\|}-\mathbf{r}_{\|}^{\prime}\right)}\left\{\Theta(z) \hat{R}(\mathbf{K}, \tilde{\mathbf{K}}) e^{i K_{z}\left(z+z^{\prime}\right)}\right\}$ is the Green's operator associated to the secondary wave reflected at the interface, and

$$
\hat{G}_{0}\left(\mathbf{r}, \mathbf{r}^{\prime}\right)=\frac{i}{8 \pi^{2}} \iint \frac{d^{2} K_{\|}}{K_{z}} e^{i K_{\|} \cdot\left(\mathbf{r}_{\|}-\mathbf{r}^{\prime} \|\right)}\left\{\Theta\left(z-z^{\prime}\right) \hat{I}(\mathbf{K}, \mathbf{K}) e^{i K_{z}\left(\mathrm{z}-z^{\prime}\right)}+\Theta\left(z^{\prime}-z\right) \hat{I}(\tilde{\mathbf{K}}, \tilde{\mathbf{K}}) e^{i K_{z}\left(z^{\prime}-z\right)}\right\}
$$

is the Green's operator associated with the primary incident wave (free-space case). Its spatial representation is $\hat{G}_{0}\left(\mathbf{r}, \mathbf{r}^{\prime}\right)=$ $\left[\hat{I}+\frac{\nabla \otimes \nabla}{k_{0}^{2}}\right] G_{0}\left(\mathbf{r}, \mathbf{r}^{\prime}\right)$ where $\hat{I}=\hat{1} \otimes \hat{1}$ is the unit dyadic and $G_{0}\left(\mathbf{r}, \mathbf{r}^{\prime}\right)=e^{i \mathbf{k}_{0}\left|\mathbf{r}-\mathbf{r}^{\prime}\right|} / 4 \pi\left|\mathbf{r}-\mathbf{r}^{\prime}\right|$ is the scalar free-space Green's function. Unlike $\hat{G}_{R}\left(\mathbf{r}, \mathbf{r}^{\prime}\right), \hat{G}_{0}\left(\mathbf{r}, \mathbf{r}^{\prime}\right)$ is singular when $\mathbf{r}=\mathbf{r}^{\prime}$. The treatment of the Green's function in the source regions has been widely discussed and is now well established [26,28,38]. When $\mathbf{r}$ is inside the volume of the scatterer, $\hat{G}_{0}\left(\mathbf{r}, \mathbf{r}^{\prime}\right)$ can be reformulated by introducing the Cauchy principal part of the integral,

$$
\begin{aligned}
\mathbf{E}^{(\mathrm{scat})}(\mathbf{r})= & \lim _{\varepsilon \rightarrow 0}\left(k_{0}^{2} \iiint_{\mathrm{Vs}-\delta V(\mathbf{r}, \varepsilon)} d^{3} r^{\prime} \eta\left(\mathbf{r}^{\prime}\right) \hat{G}_{0}\left(\mathbf{r}, \mathbf{r}^{\prime}\right) \mathbf{E}^{(\mathrm{tot})}\left(\mathbf{r}^{\prime}\right)+k_{0}^{2} \iiint_{\delta V(\mathbf{r}, \varepsilon)} d^{3} r^{\prime} \eta\left(\mathbf{r}^{\prime}\right)\left[\hat{G}_{0}\left(\mathbf{r}, \mathbf{r}^{\prime}\right) \mathbf{E}^{(\mathrm{tot})}\left(\mathbf{r}^{\prime}\right)+\hat{G}_{0}^{S}\left(\mathbf{r}, \mathbf{r}^{\prime}\right) \mathbf{E}^{(\mathrm{tot})}\left(\mathbf{r}^{\prime}\right)\right]\right. \\
& \left.+\hat{L}(\delta S(\mathbf{r}, \varepsilon)) \eta(\mathbf{r}) \mathbf{E}^{(\mathrm{tot})}(\mathbf{r})\right)
\end{aligned}
$$

where $\hat{G}_{0}^{S}\left(\mathbf{r}, \mathbf{r}^{\prime}\right)=\left[\hat{I}+\frac{\nabla \otimes \nabla}{k_{0}^{2}}\right] \frac{1}{4 \pi\left|\mathbf{r}-\mathbf{r}^{\prime}\right|}$ and $\delta V(\mathbf{r}, \varepsilon)$ is an exclusion volume containing $\mathbf{r}$ and having a chord size $\varepsilon$. The "depolarization" dyadic $\hat{L}(\delta S(\mathbf{r}, \varepsilon))=\varnothing \varnothing_{\delta S(\mathbf{r}, \varepsilon)} d^{2} r^{\prime} \frac{\hat{n}^{\prime} \otimes\left(\mathbf{r}-\mathbf{r}^{\prime}\right)}{\left|\mathbf{r}-\mathbf{r}^{\prime}\right|^{3}}$ is defined by integration on the surface $\delta S(\mathbf{r}, \varepsilon)$ enclosing $\delta V(\mathbf{r}, \varepsilon)\left(\hat{\boldsymbol{n}}^{\prime}\right.$ is an outwardly pointing unit vector normal to the surface at point $\left.\mathbf{r}^{\prime}\right)$. It depends on $\varepsilon$ and on the shape of $\delta S(\mathbf{r}, \varepsilon)$. If $\varepsilon$ is small enough, the second term on the right side of Eq. (C1) can be neglected and the regular Green's operator for the case where $\mathbf{r}, \mathbf{r}^{\prime} \in V_{S}$ can be written in the compact form

$$
\hat{G}\left(\mathbf{r}, \mathbf{r}^{\prime}\right)^{\text {in }}=\lim _{\varepsilon \rightarrow 0} \overline{\bar{G}}\left(\mathbf{r}, \mathbf{r}^{\prime}, \delta V(\mathbf{r}, \varepsilon)\right),
$$

with $\overline{\bar{G}}\left(\mathbf{r}, \mathbf{r}^{\prime}, \delta V(\mathbf{r}, \varepsilon)\right)=\hat{G}_{R}\left(\mathbf{r}, \mathbf{r}^{\prime}\right)+\left(\frac{1+\Theta\left(\left|\mathbf{r}-\mathbf{r}^{\prime}\right|-\varepsilon\right)}{2}\right) \hat{G}_{0}\left(\mathbf{r}, \mathbf{r}^{\prime}\right)+\hat{L}\left(\delta S(\mathbf{r}, \varepsilon) \frac{\delta\left(\mathbf{r}-\mathbf{r}^{\prime}\right)}{k_{0}^{2}}[\Theta\right.$ is the Heaviside function and $\delta V(\mathbf{r}, \varepsilon)$ is arbitrarily chosen as a sphere of radius $\varepsilon$ centered on $\mathbf{r}]$. In this framework the regular expression of the total electric field inside the scatterer is now $\mathbf{E}^{\text {(tot) }}(\mathbf{r})=\mathbf{E}^{\text {(inc) }}(\mathbf{r})+k_{0}^{2} \iiint_{\mathrm{Vs}_{\mathrm{s}}} d^{3} r^{\prime} \eta\left(\mathbf{r}^{\prime}\right) \hat{G}\left(\mathbf{r}, \mathbf{r}^{\prime}\right)^{\text {in }} \mathbf{E}^{\text {(tot) }}\left(\mathbf{r}^{\prime}\right)$ and is assumed to be equivalent to $\mathbf{E}^{(\mathrm{tot})}(\boldsymbol{r})=\mathbf{E}^{(\mathrm{inc})}(\mathbf{r})+\lim _{\varepsilon \rightarrow 0} k_{0}^{2} \iiint_{\mathrm{Vs}} d^{3} r^{\prime} \eta\left(\mathbf{r}^{\prime}\right) \overline{\bar{G}}\left(\mathbf{r}, \mathbf{r}^{\prime}, \delta V(\mathbf{r}, \varepsilon)\right) \mathbf{E}^{(\mathrm{tot})}\left(\mathbf{r}^{\prime}\right)$, if the limit and the integral operations can be interchanged.

Although the self-consistent development of the total field is more complicated from an analytical point of view, the discussion in Sec. III A. remains valid since the modified Green's operator $\hat{G}\left(\mathbf{r}, \mathbf{r}^{\prime}\right)^{\text {in }}$ verifies the same reciprocity property as $\hat{G}\left(\mathbf{r}, \mathbf{r}^{\prime}\right)\left[\overline{\bar{G}}\left(\mathbf{r}, \mathbf{r}^{\prime}, \delta V(\mathbf{r}, \varepsilon)\right)^{T}=\overline{\bar{G}}\left(\mathbf{r}^{\prime}, \mathbf{r}, \delta V\left(\mathbf{r}^{\prime}, \varepsilon\right)\right)\right]$. From $\hat{G}\left(\mathbf{r}, \mathbf{r}^{\prime}\right)^{\text {in }}$, it is actually possible to define a regular composed operator on the basis of Eq. (17), $\hat{\mathcal{G}}^{i}\left(\mathbf{r}, \mathbf{r}_{i}\right)^{\text {in }}=\lim _{\varepsilon \rightarrow 0} \hat{\mathcal{G}}^{i}\left(\mathbf{r}, \mathbf{r}_{i}, \delta V(\mathbf{r}, \varepsilon)\right)$ 
with

$$
\begin{aligned}
& \hat{\mathcal{G}}^{i}\left(\mathbf{r}, \mathbf{r}_{i}, \delta V(\mathbf{r}, \varepsilon)\right) \\
& \quad=\lim _{\varepsilon_{0}, \varepsilon_{1}, \ldots, \varepsilon_{i-1} \rightarrow 0}\left\{\begin{array}{c}
k_{0}^{2 i} \iiint_{\mathrm{Vs}} d^{3} r r_{0} \eta\left(\mathbf{r}_{0}\right) \iiint_{\mathrm{Vs}} d^{3} r_{1} \eta\left(\mathbf{r}_{1}\right) \iiint_{\mathrm{Vs}} d^{3} r_{2} . \eta\left(\mathbf{r}_{2}\right) \ldots \iiint_{\mathrm{Vs}^{\prime}} d^{3} r_{i-1} \eta\left(\mathbf{r}_{i-1}\right) \\
\overline{\bar{G}}\left(\mathbf{r}, \mathbf{r}_{0}, \delta \mathrm{V}(\mathbf{r}, \varepsilon) \overline{\bar{G}}\left(\mathbf{r}_{0}, \mathbf{r}_{1}, \delta \mathrm{V}\left(\mathbf{r}_{0}, \varepsilon_{0}\right) \overline{\bar{G}}\left(\mathbf{r}_{1}, \mathbf{r}_{2}, \delta \mathrm{V}\left(\mathbf{r}_{1}, \varepsilon_{1}\right) \ldots \overline{\bar{G}}\left(\mathbf{r}_{i-1}, \mathbf{r}_{i}, \delta \mathrm{V}\left(\mathbf{r}_{i-1}, \varepsilon_{i-1}\right)\right.\right.\right.\right.
\end{array}\right\} .
\end{aligned}
$$

As a consequence, all the operators defined in the discussion of Sec. III could be replaced by $\hat{\mathcal{G}}^{i}\left(\mathbf{r}, \mathbf{r}_{i}\right)^{\text {in }}$, with preserved reciprocity properties since $\hat{\mathcal{G}}^{i}\left(\mathbf{r}, \mathbf{r}_{i}\right)^{\text {in }}=\left[\hat{\mathcal{G}}^{i}\left(\mathbf{r}_{i}, \mathbf{r}\right)^{\text {in }}\right]^{\mathrm{T}}$.

\section{APPENDIX D: RECIPROCITY RELATION FOR THE GREEN'S OPERATORS}

Using the property $[\hat{U} \hat{V}]^{\mathrm{T}}=\hat{V}^{\mathrm{T}} \hat{U}^{\mathrm{T}}$, the transpose of the generalized composed Green operator of order $i>0$ given by Eq. (17) is written

$$
\hat{\mathcal{G}}^{i}\left(\mathbf{r}, \mathbf{r}_{i}\right)^{T}=k_{0}^{2 i} \iiint_{\overline{\bar{V}}_{S}} d^{3} r_{0} \eta\left(\mathbf{r}_{0}\right) \iiint_{\overline{\bar{V}}_{S}} d^{3} r_{1} \eta\left(\mathbf{r}_{1}\right) \cdots \iiint_{\overline{\bar{V}}_{S}} d^{3} \mathbf{r}_{i-1} \eta\left(\mathbf{r}_{i-1}\right) \hat{G}\left(\mathbf{r}_{i-1}, \mathbf{r}_{i}\right)^{T} \cdots \hat{G}\left(\mathbf{r}_{1}, \mathbf{r}_{2}\right)^{T} \hat{G}\left(\mathbf{r}_{0}, \mathbf{r}_{1}\right)^{T} \hat{G}\left(\mathbf{r}, \mathbf{r}_{0}\right)^{T} .
$$

On the other hand, the transposed of the elementary operator [Eq. (12)] is, for $z>0$,

$$
\hat{G}\left(\mathbf{r}, \mathbf{r}^{\prime}\right)^{T}=\frac{i}{8 \pi^{2}} \iint_{K_{x}, K_{y}} \frac{d^{2} K_{\|}}{K_{z}} e^{i \mathbf{K}_{\| \cdot} \cdot\left(\mathbf{r}_{\|}-\mathbf{r}_{\|}^{\prime}\right)}\left[\hat{R}(\mathbf{K}, \tilde{\mathbf{K}})^{T} e^{i K_{z}\left(\mathrm{z}+z^{\prime}\right)}+\Theta\left(z-z^{\prime}\right) \hat{I}(\mathbf{K}, \mathbf{K})^{T} e^{i K_{z}\left(\mathrm{z}-z^{\prime}\right)}+\Theta\left(z^{\prime}-z\right) \hat{I}(\tilde{\mathbf{K}}, \tilde{\mathbf{K}})^{T} e^{i K_{z}\left(z^{\prime}-z\right)}\right]
$$

Making the variable change $\mathbf{K}=-\tilde{\mathbf{H}}$ (or $\mathbf{H}=-\tilde{\mathbf{K}})$, the operators in Eq. (D2) are written $\hat{I}(\tilde{\mathbf{K}}, \tilde{\mathbf{K}})^{\mathrm{T}}=\hat{I}(-\mathbf{H},-\mathbf{H})^{\mathrm{T}}=$ $\hat{I}(-\mathbf{H},-\mathbf{H})=\hat{I}(\mathbf{H}, \mathbf{H}), \hat{I}(\mathbf{K}, \mathbf{K})^{\mathrm{T}}=\hat{I}(-\tilde{\mathbf{H}},-\tilde{\mathbf{H}})^{\mathrm{T}}=\hat{I}(-\tilde{\mathbf{H}},-\tilde{\mathbf{H}})=\hat{I}(\tilde{\mathbf{H}}, \tilde{\mathbf{H}})$,

and

$$
\begin{aligned}
\hat{R}(\mathbf{K}, \tilde{\mathbf{K}}) & =\hat{R}(-\tilde{\mathbf{H}},-\mathbf{H})=r_{s}\left(-H_{\|}\right) \hat{\boldsymbol{s}}(-\tilde{\mathbf{H}}) \otimes \hat{\boldsymbol{s}}(-\mathbf{H})+r_{p}\left(-H_{\|}\right) \hat{\boldsymbol{p}}(-\tilde{\mathbf{H}}) \otimes \hat{\boldsymbol{p}}(-\mathbf{H}) \\
& =r_{s}\left(H_{\|}\right) \hat{\boldsymbol{s}}(\tilde{\mathbf{H}}) \otimes \hat{\boldsymbol{s}}(\mathbf{H})+r_{p}\left(H_{\|}\right) \hat{\boldsymbol{p}}(\tilde{\mathbf{H}}) \otimes \hat{\boldsymbol{p}}(\mathbf{H})=\hat{R}(\mathbf{H}, \tilde{\mathbf{H}})^{T}
\end{aligned}
$$

Since the integration in Eq. (D2) runs over all possible values of $H_{x}$ and $H_{y}$ from $-\infty$ to $+\infty$ and $-\tilde{\mathbf{H}}_{\|}=-\mathbf{H}_{\|}$, it can be found that

$$
\hat{G}\left(\mathbf{r}, \mathbf{r}^{\prime}\right)^{T}=\hat{G}\left(\mathbf{r}^{\prime}, \mathbf{r}\right)
$$

and, finally, from Eqs. (D1) and (D3),

$$
\hat{\mathcal{G}}^{i}\left(\mathbf{r}, \mathbf{r}_{i}\right)^{T}=\hat{\mathcal{G}}^{i}\left(\mathbf{r}_{i}, \mathbf{r}\right)
$$

[1] C. F. Bohren and D. P. Huffman, Absorption and Scattering of Light by Small Particles (Wiley, New York, 1983).

[2] H. C. van de Hulst, Light Scattering by Small Particles (Dover Publications Inc., New York, 1981).

[3] D. R. Lytle, II, P. S. Carney, J. C. Schotland, and E. Wolf, Phys. Rev. E 71, 056610 (2005).

[4] N. Dahan and J. J. Greffet, Opt. Express 20, A530 (2012).

[5] A. Small, J. Fung, and V. N. Manoharan, J. Opt. Soc. Am. A 30, 2519 (2013).

[6] D. Torrungrueng, B. Ungan, and J. T. Johnson, IEEE Geosci. Remote Sens. Lett. 1, 131 (2004).

[7] M. Pellarin et al., J. Phys. Chem. C 123, 15217 (2019).

[8] H. von Helmholtz, Handbuch der Physiologischen Optik, 3rd ed. (Leopold Voss, Hamburg\& Leipzig, 1909), pp. 198-199.

[9] F. J. J. Clarke and D. J. Parry, Lighting Res. Technol. 17, 1 (1985).

[10] R. J. Potton, Rep. Prog. Phys. 67, 717 (2004).

[11] A. L. Shelankov and G. E. Pikus, Phys. Rev. B 46, 3326 (1992).
[12] M. Iwanaga, A. S. Vengurlekar, T. Hatano, and T. Ishihara, Am. J. Phys. 75, 899 (2007).

[13] M. Mansuripur and D. P. Tsai, Opt. Commun. 284, 707 (2011).

[14] R. Carminati, M. Neito-Vesperinas, and J. J. Greffet, J. Opt. Soc. Am. A 15, 706 (1998).

[15] M. A. Dupertuis, B. Acklin, and M. Proctor, J. Opt. Soc. Am. A 11, 1167 (1994).

[16] C. G. A. Drezet, in Singular and Chiral Nanoplasmonics, edited by N. I. Z. Svetlana and V. Boriskina (Pan Stanford Publishing Pte. Ltd., Singapore, 2015), p. 57.

[17] A. T. de Hoop, Appl. Sci. Res. B 8, 135 (1959).

[18] M. Born and E. Wolf, Principles of Optics: Electromagnetic Theory of Propagation Interference and Diffraction of Light, 7th ed. (Cambridge Univerity Press, Cambridge, 1999).

[19] S. K. Cho, Electromagnetic Scattering (Springer, New York, 1990), p. 93.

[20] H. Lorentz, Collected Papers (Martinus Nijhoff, Den Haag, 1936), Vol. 3, pp. 1-11. 
[21] L. Novotny and B. Hecht, Principles of Nano-Optics (Cambridge University Press, New York, 2006).

[22] T. M. Søndergaard, Green's Function Integral Equation Methods in Nano-Optics (CRC Press, Taylor \& Francis, Boca Raton, 2019).

[23] A. A. Maradudin and D. L. Mills, Phys. Rev. B 11, 1392 (1975).

[24] A. Pors and S. I. Bozhevolnyi, ACS Photonics 2, 228 (2015).

[25] J. E. Sipe, J. Opt. Soc. Am. B 4, 481 (1987).

[26] S. Albaladejo, R. Gomez-Medina, L. S. Froufe-Perez, H. Marinchio, R. Carminati, J. F. Torrado, G. Armelles, A. GarciaMartin, and J. J. Saenz, Opt. Express 18, 3556 (2010).

[27] A. Lakhtakia, Int. J. Mod. Phys. C 03, 583 (1992).

[28] A. D. Yaghjian, Proceedings of the IEEE 68, 248 (1980).

[29] L. D. Landau, E. M. Lifshitz, and L. P. Pitaevskii, Electrodynamics of Continuous Media, 2nd ed. (Pergamon Press, Oxford, 1984).

[30] N. P. Armitage, Phys. Rev. B 90, 035135 (2014).
[31] P. B. Johnson and R. W. Christy, Phys. Rev. B 6, 4370 (1972).

[32] J. J. Yang, M. Perrin, and P. Lalanne, Phys. Rev. X 5, 021008 (2015).

[33] M. Decker, M. W. Klein, M. Wegener, and S. Linden, Opt. Lett. 32, 856 (2007).

[34] A. Drezet, C. Genet, J. Y. Laluet, and T. W. Ebbesen, Opt. Express 16, 12559 (2008).

[35] V. A. Fedotov, P. L. Mladyonov, S. L. Prosvirnin, A. V. Rogacheva, Y. Chen, and N. I. Zheludev, Phys. Rev. Lett. 97, 167401 (2006).

[36] M. Kuwata-Gonokami, N. Saito, Y. Ino, M. Kauranen, K. Jefimovs, T. Vallius, J. Turunen, and Y. Svirko, Phys. Rev. Lett. 95, 227401 (2005).

[37] E. Vinegrad, D. Vestler, A. Ben-Moshe, A. R. Barnea, G. Markovich, and O. Cheshnovsky, ACS Photonics 5, 2151 (2018).

[38] M. A. Yurkin and A. G. Hoekstra, J. Quant. Spectrosc. Ra. 106, 558 (2007). 